\title{
Linked vortices as baryons in the miscible BEC-Skyrme model
}

\author{
Sven Bjarke Gudnason๑* \\ Institute of Contemporary Mathematics, School of Mathematics and Statistics, Henan University, \\ Kaifeng, Henan 475004, People's Republic of China \\ Muneto $\mathrm{Nitta}^{\dagger}$ \\ Department of Physics, and Research and Education Center for Natural Sciences, Keio University, \\ Hiyoshi 4-1-1, Yokohama, Kanagawa 223-8521, Japan
}

(Received 10 June 2020; accepted 14 August 2020; published 31 August 2020)

\begin{abstract}
We introduce a variation of the Bose-Einstein condensate(BEC)-Skyrme model, with an altered potential for miscible BECs that gives rise to two physical vortex strings. In the ground state of each topological sector, the vortices are linked exactly $B$ times, due to a recently formulated theorem, with $B$ being the baryon number of the solution. The model also possesses metastable states, where the vortices are degenerate and do not lend the interpretation of the baryon number as the linking number of the vortices.
\end{abstract}

DOI: $10.1103 /$ PhysRevD.102.045022

\section{INTRODUCTION}

More than a century ago, Lord Kelvin imagined that atoms were made of knotted vortices [1], but this idea has not been successful so far. However, recently we have made a connection between not knots, but links of vortices and Skyrmions in the Skyrme model [2]. Skyrmions are solitons of the texture type in 3-dimensional space and possess a topological degree $B$ as they are mapped to a 3 -sphere, being the target space or isospin space $[3,4]$. In the large- $N$ limit of QCD, the baryon is identified with the Skyrmion [5,6], thus providing a solitonic approach to nuclear physics, see, e.g., Refs. [7-9]. The Skyrmion may also be realized in two-component Bose-Einstein condensates (BECs) [10-20], see Ref. [21] for a review. In Refs. [22-24] we introduced a potential inspired by twocomponent BECs, $V \sim M^{2}\left|\phi_{1}\right|^{2}\left|\phi_{2}\right|^{2}$, which deforms the Skyrmions into a twisted vortex ring (or vorton) by explicitly breaking the $\mathrm{SU}(2)$ isospin symmetry normally possessed by Skyrmion solutions. Due to the nonlinear sigma model constraint, $\left|\phi_{1}\right|^{2}+\left|\phi_{2}\right|^{2}=1$, the center of the vortex ring in one component confines the other component; it is thus a global analog of Witten's superconducting cosmic string [25]. Similar vortex rings can also be obtained in a different asymmetric potential,

\footnotetext{
*gudnason@henu.edu.cn

nitta@phys-h.keio.ac.jp
}

Published by the American Physical Society under the terms of the Creative Commons Attribution 4.0 International license. Further distribution of this work must maintain attribution to the author(s) and the published article's title, journal citation, and DOI. Funded by SCOAP.
$V \sim M^{2}\left|\phi_{1}\right|^{2}$ [26]. In addition to a vortex ring, the BECinspired potential also allows for a domain wall [22-24] into which the vortex ring can be absorbed, creating a vortex handle - or rather a link of a handle and a dual handle from both sides of the domain wall [27]. We would like to stress that the Skyrmions we study here are closer to those describing nuclei within the Skyrme model, than to the Skyrmions in BEC, because of the derivative part of the Lagrangian. The potential and the terminology is borrowed from BECs and hence similar behavior is expected.

The connection between links of vortices and Skyrmions [2] is made by means of a theorem stating that there exists a projection of the Skyrmion field onto a 2-sphere (as opposed to the 3-sphere that is the target space), which has the properties that two distinct regular points are linked $B$ times in real physical 3-space, with $B$ being the baryon number or topological degree of the Skyrmion. Taking a natural Ansatz for the Skyrmion, like the one giving rise to a $B$-twisted vortex ring [22-24], produces a "physical" vortex with winding number one and a "vacuum" vortex with winding number $B$. The "vacuum" vortex is not physical in the sense that it strays off to infinity in such a diluted form that the total energy is finite. As mentioned, the catch is that the points under the projection have to be regular points, which is not always the case. In Ref. [2] we have circumvented the issue by introducing a rotation of the 2-sphere, hence making it possible to find points which are regular and hence giving rise to nondegenerate vortices that thus provide the linking number $Q=B$. One may consider such a rotation a bit arbitrary and thus wonder about the physical implication thereof.

In this paper, we modify the BEC-inspired potential studied in Refs. [22-24,27] by flipping the sign of the 
potential (which is allowed in a nonlinear sigma model) and this is suitable for the class of BECs called miscible twocomponent BECs, for which both the components $\phi_{1}$ and $\phi_{2}$ develop vacuum expectation values (VEVs) in the vacua. The implications thereof is a rather crucial change in the vacuum structure and indeed the model only possesses a unique vacuum state after the altercation, which we dub the miscible BEC-Skyrme model. This case admits two kinds of vortices having windings in the $\phi_{1}$ and $\phi_{2}$ components as in the case of the miscible BEC $[21,28,29]$. As a consequence, the above-mentioned "vacuum" vortex is transformed into a second "physical" vortex, thus realizing, in a physical way, the two linked vortices proposed in Ref. [2].

Moreover there is the issue of regular points leading to nondegenerate links of vortices versus singular points leading to degenerate vortices in the framework of Ref. [2]. This notion takes a very physical form in the presence of the miscible BEC-inspired potential, as the degenerate vortex links generally give rise to higher-energy (metastable) states and the nondegenerate vortex links yield stable (ground) states.

This paper is organized as follows. In Sec. II we introduce the miscible BEC-Skyrme model and discuss the vacuum structure of the model. In Sec. III we define the numerical observables, explain the numerical method, define the initial conditions used throughout the paper and discuss the numerical results. Finally, we conclude the paper with a discussion in Sec. IV.

\section{THE MISCIBLE BEC-SKYRME MODEL}

We consider the generalized Skyrme model which contains the kinetic term, the Skyrme term $[3,4]$ and the BPS-Skyrme (Bogomol'nyi-Prasad-Sommerfield-Skyrme) term $[30-32]^{1}$ as well as a potential

$$
\begin{gathered}
\mathcal{L}=\mathcal{L}_{2}+c_{4} \mathcal{L}_{4}+c_{6} \mathcal{L}_{6}-V, \\
\mathcal{L}_{2}=-\frac{1}{2} \partial_{\mu} \boldsymbol{\phi}^{\dagger} \partial^{\mu} \boldsymbol{\phi}, \\
\mathcal{L}_{4}=\frac{1}{8}\left(\partial_{\mu} \boldsymbol{\phi}^{\dagger} \partial_{\nu} \boldsymbol{\phi}\right)\left(\partial^{[\mu} \boldsymbol{\phi}^{\dagger} \partial^{\nu]} \boldsymbol{\phi}\right) \\
+\frac{1}{8}\left(\partial_{\mu} \boldsymbol{\phi}^{\dagger} \sigma^{2} \partial_{\nu} \boldsymbol{\phi}\right)\left(\partial^{[\mu} \boldsymbol{\phi}^{\dagger} \sigma^{2} \partial^{\nu]} \boldsymbol{\phi}\right) \\
=-\frac{1}{4}\left(\partial_{\mu} \boldsymbol{\phi}^{\dagger} \partial^{\mu} \boldsymbol{\phi}\right)^{2}+\frac{1}{16}\left(\partial_{\mu} \boldsymbol{\phi}^{\dagger} \partial_{\nu} \boldsymbol{\phi}+\partial_{\nu} \boldsymbol{\phi}^{\dagger} \partial_{\mu} \boldsymbol{\phi}\right)^{2}, \\
\mathcal{L}_{6}=\frac{1}{4}\left(\epsilon^{\mu \nu \rho \sigma} \boldsymbol{\phi}^{\dagger} \partial_{\nu} \boldsymbol{\phi} \partial_{\rho} \boldsymbol{\phi}^{\dagger} \partial_{\sigma} \boldsymbol{\phi}\right)^{2},
\end{gathered}
$$

\footnotetext{
${ }^{1}$ The BPS-Skyrme term is named after the BPS-Skyrme model of Refs. [31,32], because it possesses a saturable energy bound (BPS bound); this model consists only of the BPS-Skyrme term and a potential.
}

where $\sigma^{a}$ are the Pauli matrices, $\boldsymbol{\phi} \equiv\left(\phi_{1}(x), \phi_{2}(x)\right)^{\mathrm{T}}$ is a complex two-vector of scalar fields which obey the nonlinear sigma model constraint $\boldsymbol{\phi}^{\dagger} \boldsymbol{\phi}=\left|\phi_{1}\right|^{2}+\left|\phi_{2}\right|^{2}$, the spacetime indices $\mu, \nu, \rho, \sigma$ run over 0 through 3 and the flat Minkowski metric is taken to be of the mostly positive signature. The relation between the complex 2-vector of scalar fields, $\boldsymbol{\phi}$, and the chiral Lagrangian field usually used in Skyrme-type models is given by

$$
U=\left(\begin{array}{ll}
\boldsymbol{\phi} & -\mathrm{i} \sigma^{2} \overline{\boldsymbol{\phi}}
\end{array}\right)=\left(\begin{array}{cc}
\phi_{1} & -\bar{\phi}_{2} \\
\phi_{2} & \bar{\phi}_{1}
\end{array}\right)
$$

which translates the constraint $\boldsymbol{\phi}^{\dagger} \boldsymbol{\phi}=\operatorname{det} U=1$ into the usual one in terms of $U$.

The Skyrme term (3) consists of a particular combination of two operators in the chiral Lagrangian, where the coefficients are tuned so that the fourth-order time derivative of $U$ cancels out, see for example Ref. [33] for details. The BPS-Skyrme term (4), likewise is a particular combination of three operators in the chiral Lagrangian at order $p^{6}$ (six derivatives), where again the coefficients are tuned so that there is only a second-order time derivative of $U$, but no fourth order or sixth order ones (see Ref. [33]).

With the potential turned off, the target space manifold is given by $\mathrm{O}(4) / \mathrm{O}(3) \simeq \mathrm{SU}(2) \simeq S^{3}$ which is a unit 3 -sphere. This is due to the model without a potential possessing $\mathrm{O}(4)$ symmetry, which however would spontaneously break to $\mathrm{O}(3)$ by the requirement of finite energy. The maps with finite energy necessarily have vanishing derivatives at spatial infinity, which effectively point compactifies $\mathbb{R}^{3}$ to $\mathbb{R}^{3} \cup\{\infty\} \simeq S^{3}$. Hence, topological solitons are supported with topological degree $B \in \pi_{3}\left(S^{3}\right)=\mathbb{Z}$, where $B$ is given by

$$
B=\frac{1}{4 \pi^{2}} \int \mathrm{d}^{3} x \mathcal{B}, \quad \mathcal{B} \equiv \epsilon^{i j k} \boldsymbol{\phi}^{\dagger} \partial_{i} \boldsymbol{\phi} \partial_{j} \boldsymbol{\phi}^{\dagger} \partial_{k} \boldsymbol{\phi},
$$

where $i, j, k=1,2,3$ are spatial indices.

In this paper we will augment the model with the miscible BEC-inspired potential

$$
V=\frac{1}{8} M^{2}\left[\left(\boldsymbol{\phi}^{\dagger} \sigma^{3} \boldsymbol{\phi}\right)^{2}-1\right]=-\frac{1}{2} M^{2}\left|\phi_{1}\right|^{2}\left|\phi_{2}\right|^{2},
$$

which is the same as the BEC-inspired potential used in Refs. [22-24], however with $M^{2} \rightarrow-M^{2}$. The previous and current ones are called immiscible and miscible, respectively, in two-component BECs. This change of the sign of the potential has crucial influence on the vacuum structure since the vacuum now breaks the continuous symmetry of the model completely; therefore both complex scalars gain VEVs and describe a miscible BEC phase.

Turning on the potential (7) explicitly breaks the symmetry from $\mathrm{O}(4)$ to 


$$
G=\mathrm{U}(1) \times \mathrm{O}(2) \simeq \mathrm{U}(1)_{0} \times\left[\mathrm{U}(1)_{3} \rtimes\left(\mathbb{Z}_{2}\right)_{1,2}\right],
$$

where the group actions defined by the above symmetries are given by

$$
\begin{gathered}
\mathrm{U}(1)_{0}: \boldsymbol{\phi} \rightarrow e^{\mathrm{i} \omega} \boldsymbol{\phi}, \\
\mathrm{U}(1)_{3}: \boldsymbol{\phi} \rightarrow e^{\mathrm{i} \eta \sigma^{3}} \boldsymbol{\phi}, \\
\left(\mathbb{Z}_{2}\right)_{1,2}: \boldsymbol{\phi} \rightarrow e^{\mathrm{i} \frac{\pi}{2}\left(a \sigma^{1}+b \sigma^{2}\right)} \boldsymbol{\phi},
\end{gathered}
$$

with $a^{2}+b^{2}=1$. The $\left(\mathbb{Z}_{2}\right)_{1,2}$ exchanges $\phi_{1}$ and $\phi_{2}$ as

$$
\left(\phi_{1}, \phi_{2}\right)^{\mathrm{T}} \rightarrow\left(e^{\mathrm{i} \gamma} \phi_{2}, e^{-\mathrm{i} \gamma} \phi_{1}\right)^{\mathrm{T}},
$$

with $e^{\mathrm{i} \gamma}=b+\mathrm{i} a \cdot \mathrm{U}(1)_{3}$ is acting on $\left(\mathbb{Z}_{2}\right)_{1,2}$ transforming $\gamma$ such that they define a semidirect product which we have denoted by $\rtimes$.

In stark contrast to the immiscible BEC-inspired potential, the miscible version accommodates only a connected vacuum state

$$
\boldsymbol{\phi}_{\mathrm{vac}}=\frac{1}{\sqrt{2}}\left(e^{\mathrm{i} \alpha}, e^{\mathrm{i} \beta}\right)^{\mathrm{T}},
$$

spontaneously breaking the symmetry $G$ into a $\mathbb{Z}_{2}$ subgroup (12) with

$$
\gamma=\alpha-\beta,
$$

which we denote by $\left(\mathbb{Z}_{2}\right)_{\alpha-\beta}$. The target space is thus given by

$\mathcal{M}=G / H=\left[\mathrm{U}(1)_{0} \times \mathrm{O}(2)\right] /\left(\mathbb{Z}_{2}\right)_{\alpha-\beta}=U(1)_{1} \times U(1)_{2}$,

which does not allow for domain walls because of

$$
\pi_{0}(\mathcal{M})=\mathbf{1},
$$

but accommodates two types of vortices supported by

$$
\pi_{1}(\mathcal{M})=(\mathbb{Z})_{1} \times(\mathbb{Z})_{2}
$$

as the case of miscible two-component BECs [21,28,29].

The absence of the domain wall is in contrast to the case of immiscible BEC-Skyrme model with the spontaneously breaking of $\left(\mathbb{Z}_{2}\right)_{1,2}$ admitting a domain wall. The immiscible BEC-Skyrme model was shown to contain vortex rings in Ref. [27] and when pushed toward a domain wallpresent for the immiscible BEC-inspired potential-the vortex ring turned into a handle (in say $\phi_{1}$ ) on the domain wall, albeit creating a second dual handle (in $\phi_{2}$ ). The two seemingly different vortices were always present at the same time and hence topologically the immiscible BECinspired potential of Ref. [27] gives rise to a symmetry breaking supporting only one topological number for the vortices. In principle, in this model we can have two independent numbers of vortices.

The parameter space of the model with $c_{4}, c_{6}$, and $M$ is rather large and for this reason we will choose only two model points

$$
\begin{aligned}
& 2+4 \text { model }: c_{4}=1, \quad c_{6}=0, \\
& 2+6 \text { model }: c_{4}=0, \quad c_{6}=1,
\end{aligned}
$$

which disentangles the effects of the Skyrme term $\left(c_{4} \neq 0\right)$ and the BPS-Skyrme term $\left(c_{6} \neq 0\right)$.

\section{SKYRMIONS AS LINKED VORTICES}

\section{A. Local observables}

In order to observe the linked vortices in Skyrmions we need some observables to identify the vortices. We will consider the baryon charge density $\mathcal{B}$, the static energy density $\mathcal{E}=-\mathcal{L}$, the potential energy (density) $V$ as well as a vorticity density. In order to define the latter, we will construct a term inspired by BPS vortices in the AbelianHiggs model at critical coupling. Starting with the BPS equation

$$
\begin{gathered}
F_{12}=e^{2}\left(|\phi|^{2}-v^{2}\right), \\
D_{\bar{z}} \phi=0,
\end{gathered}
$$

and aiming at constructing an expression for the topological vortex charge

$$
Q=\frac{1}{2 \pi} \int \mathrm{d}^{2} x F_{12}
$$

we can solve for the gauge field from Eq. (21) obtaining

$$
A_{\bar{z}}=-\mathrm{i} \partial_{\bar{z}} \log \phi,
$$

from which we can readily construct $F_{12}$ :

$F_{12}=-2 \mathrm{i} F_{z \bar{z}}=-2 \partial_{z} \partial_{\bar{z}} \log |\phi|^{2}=-\frac{1}{2}\left(\partial_{1}^{2}+\partial_{2}^{2}\right) \log |\phi|^{2}$,

which is an expression for the topological vortex charge density for a vortex pointing in the $x^{3}$ direction. A simple extension of the charge density to cover a vortex pointing in any direction in $\mathbb{R}^{3}$ can thus be made as

$$
\mathcal{Q}_{1,2}=\frac{1}{2} \sum_{i} \epsilon_{i j k} F_{j k}=-\left(\partial_{1}^{2}+\partial_{2}^{2}+\partial_{3}^{2}\right) \log \left|\phi_{1,2}\right|^{2},
$$


where we have added a label for the two complex fields in our model.

For definiteness, we will define the total energy by the integral expression

$$
E_{B}=\int \mathrm{d}^{3} x \mathcal{E}=-\int \mathrm{d}^{3} x \mathcal{L}
$$

where the subscript $B$ labels the topological sector at hand.

\section{B. Numerical method}

In order to explore the model, we need to find numerical solutions to the equations of motion, which we will solve by using the arrested Newton flow method [34] for a cubic $100^{3}$ lattice, where the spatial derivatives are discretized using a standard finite difference scheme with a fourthorder 5-point stencil. The arrested Newton flow is updated in the "time direction" using a fourth-order Runge-Kutta method.

\section{Initial conditions}

In this paper, the linked vortices will naturally be physical due to the potential (7). Nevertheless, it is not a priori guaranteed that the linked vortices are not degenerate, obscuring the interpretation that the linking number is equal to the topological degree of the Skyrmions, as proved by the theorem given in Ref. [2]. One could expect that increasing the coupling of the potential $M^{2}$, would eventually force the linked vortices to be nondegenerate. This will be investigated numerically in the next subsection.

As initial conditions we use both the rational map approximations of Ref. [35], whose linked vortices were interpreted and studied in Ref. [2], and composite Skyrmions constructed from lower-charge solutions using the symmetric product Ansatz

$$
U^{\text {prod }}=\frac{U_{1} U_{2}+U_{2} U_{1}}{\operatorname{det}\left(U_{1} U_{2}+U_{2} U_{1}\right)} .
$$

As already mentioned in the introduction, the linked vortices in Ref. [2] naturally contain a vacuum vortex, which is not particularly physical, because it extends to infinity in $\mathbb{R}^{3}$ although its flux is diluted such that the energy is finite. In Ref. [2] we introduced a rotation of the 2-sphere after applying the Hopf map to a Skyrmion map, which effectively eliminated the degeneracy of the linked vortices, allowing for the interpretation of the linking number as the topological charge or baryon number of the Skyrmions.

In this paper, we do not need to perform such a rotation of the 2-sphere, but it is necessary to rotate the standard frame of a Skyrmion such that the vacuum is compatible with the potential (7) used here. That is, a normal Skyrmion is a map $\phi: \mathbb{R}^{3} \rightarrow S^{3}$ with the vacuum chosen to be

$$
U_{\text {vac }}^{\text {standard frame }}=\mathbf{1}_{2},
$$

which translates to the vacuum in $\phi$ :

$$
\boldsymbol{\phi}_{\mathrm{vac}}^{\text {standard frame }}=\left(\begin{array}{l}
1 \\
0
\end{array}\right),
$$

which is obviously not the vacuum (13) in this model, i.e., with the potential (7), but is indeed compatible with a normal pion mass term $\operatorname{tr}\left(\mathbf{1}_{2}-U\right)$ or any generalization thereof. Therefore, for all initial conditions, which are constructed to match the standard frame, we perform the following rotation

$$
\tilde{\phi}=\frac{1}{\sqrt{2}}\left(\begin{array}{cc}
1 & 1 \\
-1 & 1
\end{array}\right) \Re(\boldsymbol{\phi})+\Im(\boldsymbol{\phi}),
$$

which is an $\mathrm{SO}(4)$ transformation transforming the standard frame vacuum (29) into

$$
\tilde{\phi}_{\mathrm{vac}}=\frac{1}{\sqrt{2}}\left(\begin{array}{c}
1 \\
-1
\end{array}\right) .
$$

This corresponds to the vacuum of this model in Eq. (13) with $\alpha=0$ and $\beta=\pi$.

The initial conditions are now ready to be studied using the numerical method described in the previous subsection and the results will be given next.

\section{Numerical results}

We are now ready to present the numerical results in the $2+4$ and $2+6$ models for various values of $M$, with $M^{2}$ the coefficient of the miscible BEC-inspired potential.

Starting with the topological sector $B=1$, the soliton solution called the Skyrmion, is spherically symmetric without the potential (i.e., for $M=0$ ). The spherical symmetry means that a spatial $\mathrm{SO}(3)$ rotation can be undone by an $\mathrm{SU}(2)$ isospin (internal) rotation. Once $M \neq 0$ is turned on, the spherical symmetry is explicitly broken. The numerical results for $B=1$ with $M=0,1, \ldots, 7$ are shown Fig. 1 for both (a) the $2+4$ model and (b) the $2+6$ model. This figure and all the remaining figures of the same type in this paper are composed of 4 columns showing isosurfaces of the topological baryon charge density, of the total energy density, of the potential and of the vorticities defined in Eq. (25), respectively. The color scheme utilized in the first 3 columns is the one used for standard Skyrmions, where the three real components $\Im \phi_{1}$, $\Re \phi_{2}$ and $\Im \phi_{2}$ are normalized

$$
\left(\begin{array}{l}
n_{1} \\
n_{2} \\
n_{3}
\end{array}\right) \equiv \frac{1}{\left(\Im \phi_{1}\right)^{2}+\left|\phi_{2}\right|^{2}}\left(\begin{array}{c}
\Im \phi_{1} \\
\Re \phi_{2} \\
\Im \phi_{2}
\end{array}\right),
$$




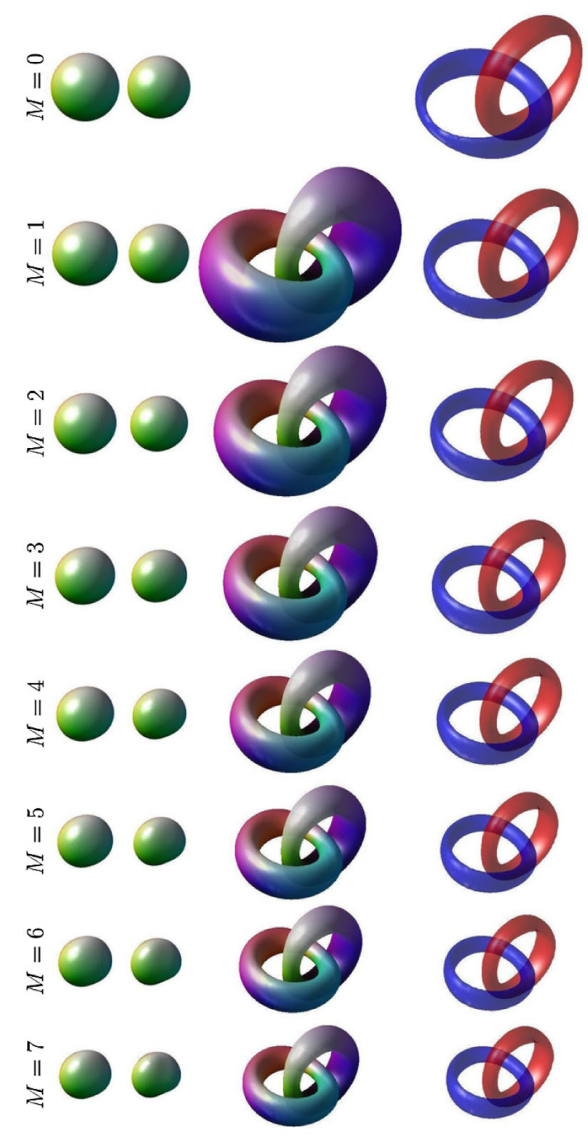

(a)

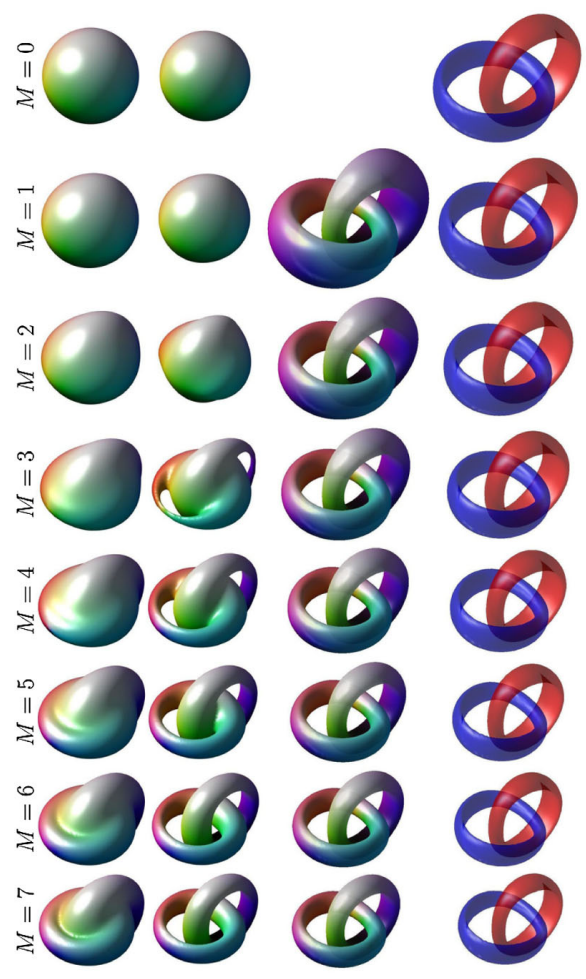

(b)

FIG. 1. The $B=1$ Skyrmion in the miscible BEC-Skyrme (a) $2+4$ model (b) $2+6$ model. The four columns show isosurfaces of: the topological baryon charge density $\mathcal{B}$, the energy density $\mathcal{E}$, the potential $V$, and the vorticities $\mathcal{Q}_{1,2}$ in $\phi_{1,2}$ (with red and blue, respectively). The color scheme used for the first 3 columns is described in the text. Each row corresponds to a different value of the potential parameter $M^{2}$.

where $n_{1}+\mathrm{i} n_{2}=\exp (\mathrm{i} H)$ is mapped to the hue $H$ and $n_{3}$ determines the lightness, such that $n_{3}=1$ is white, $n_{3}=-1$ is black and $n_{3}=0$ is a color determined by $H: H=0$ is red, $H=2 \pi / 3$ is green and $H=4 \pi / 3$ is blue. The intent is simply to show how the surfaces are mapped to the target space $S^{3}$. The last column in each panel of Fig. 1 shows the vorticities given in Eq. (25) such that the vorticity density in $\phi_{1}\left(\phi_{2}\right)$ is given by $Q_{1}\left(Q_{2}\right)$ and shown with red (blue) surfaces. All the isosurfaces are shown at half-maximum of the corresponding observable, except for the vorticities, which are shown at a quarter of the maximum vorticity.

Figure 1(a) shows the $B=1$ Skyrmion for various values of $M$ in the $2+4$ model. It is interesting to note that the vorticities in the 4th column are present with the potential turned off $(M=0){ }^{2}$ Although both the potential (3rd column) and the vorticities clearly show

\footnotetext{
${ }^{2}$ The standard frame would make one of the vortices a "vacuum" vortex and the other a "physical" vortex, see Ref. [2]. The transformation (30) is thus equivalent to one of the rotations of the 2-sphere performed in Ref. [2].
}

the 1-Skyrmion contains a pair of linked vortices for $M>0$, and hence with linking number 1 -in accord with the theorem of Ref. [2], the linked vortices do not quite show themselves in the topological baryon charge density or the total energy density for the $2+4$ model [Fig. 1(a)]. All that happens is that the baryon charge density and energy density isosurfaces are slightly deformed, which is expected due to the broken $\mathrm{SU}(2)$ symmetry.

Figure 1(b) shows the $B=1$ Skyrmion for various values in the $2+6$ model. In comparison with the $2+4$ model, the total energy density isosurface (at halfmaximum density) is quickly transformed from a sphere into the shape given by two linked vortices, see the 2nd column of Fig. 1(b) - this happens around $M \lesssim 3$. To some extent the linked vortices also become visible in the baryon charge density, see the first column of the figure.

We now turn to the case of the $B=2$ Skyrmion, which without a potential is torus shaped. Figures $2(\mathrm{a})$ and $2(\mathrm{~b})$ show the results for various masses $M$ in the $2+4$ model and $2+6$ model, respectively. More precisely, we start with the standard $B=2$ Skyrmion transformed by Eq. (30) 
(a)
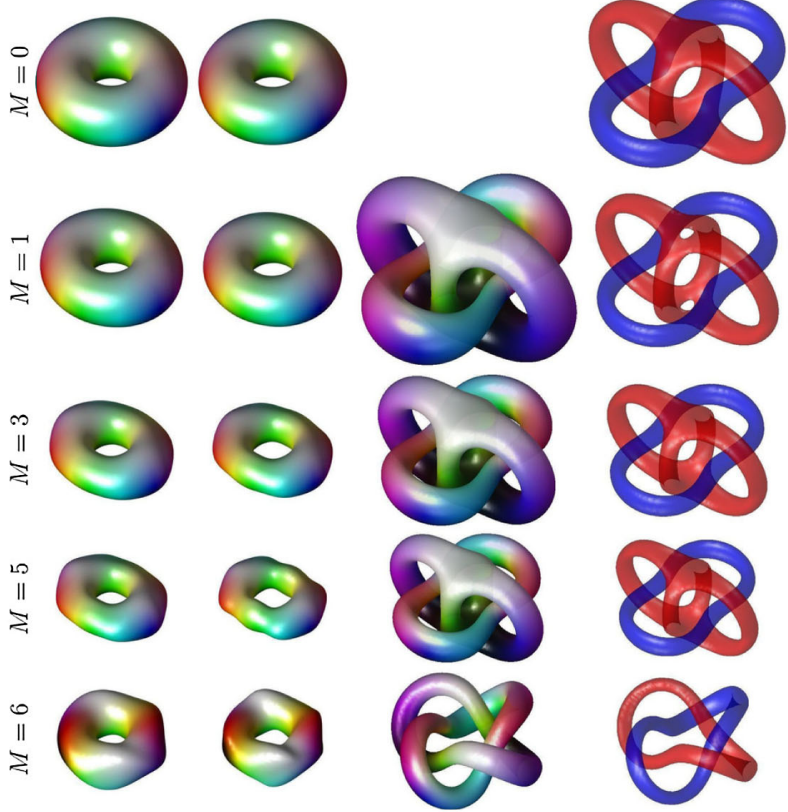

(b)
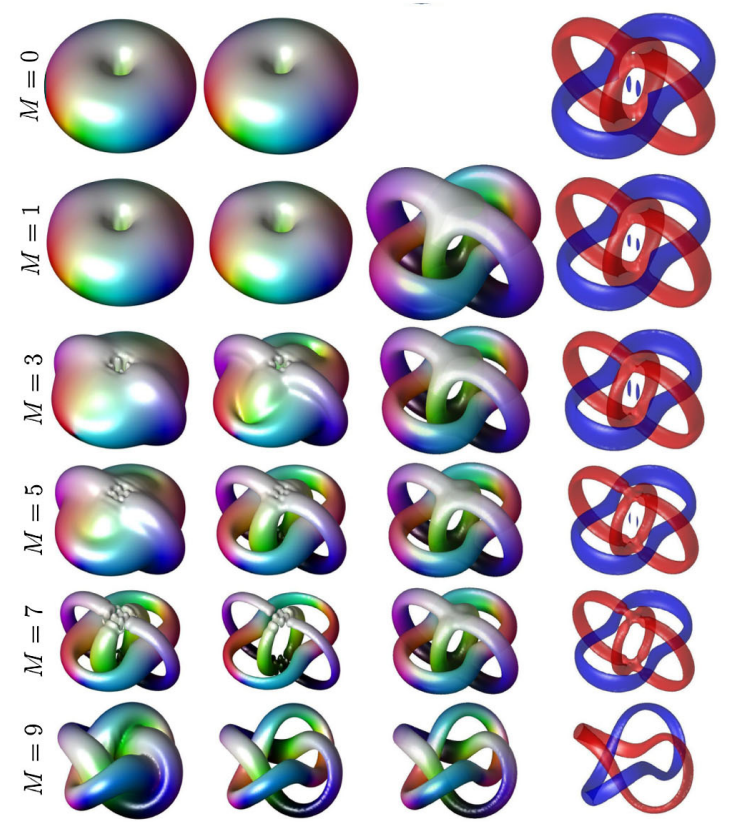

FIG. 2. The metastable $B=2$ Skyrmion in the miscible BECSkyrme (a) $2+4$ model (b) $2+6$ model. The four columns show isosurfaces of: the topological baryon charge density $\mathcal{B}$, the energy density $\mathcal{E}$, the potential $V$, and the vorticities $\mathcal{Q}_{1,2}$ in $\phi_{1,2}$ (with red and blue, respectively). The color scheme used for the first 3 columns is described in the text. Each row corresponds to a different value of the potential parameter $M^{2}$.

as the initial guess. We notice that the vorticities (4th column) are degenerate even for $M=0$ and so is the potential energy ( 3 rd column) once turning on a finite potential, $M=1$. In order to define what we mean by degenerate, let us make the following definition: (a)
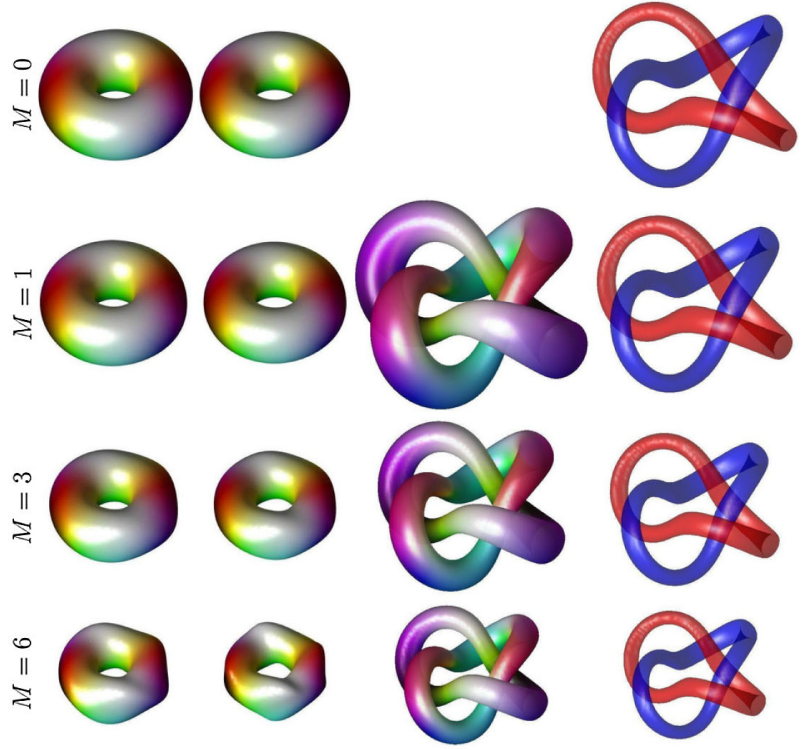

(b)
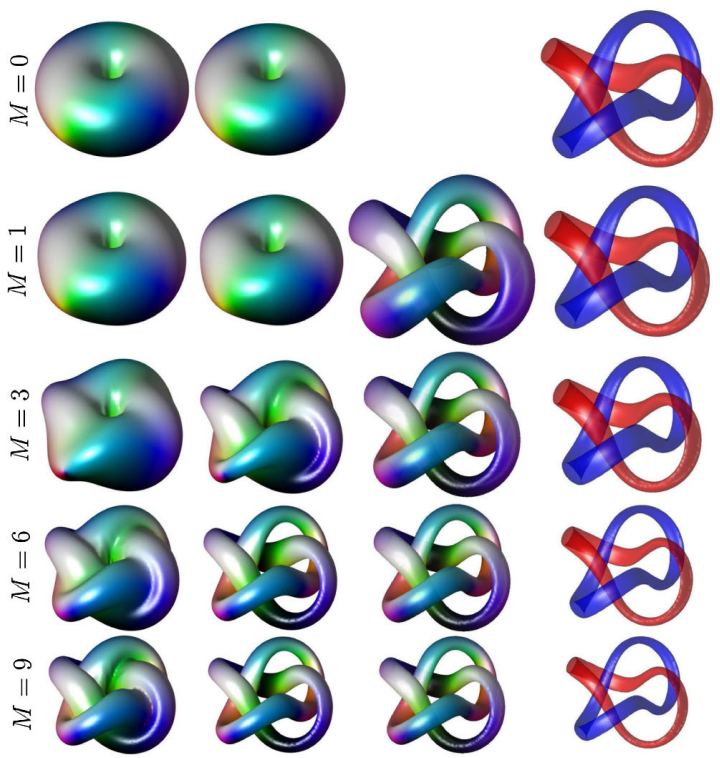

FIG. 3. The stable $B=2$ Skyrmion in the miscible BECSkyrme (a) $2+4$ model (b) $2+6$ model. The four columns show isosurfaces of: the topological baryon charge density $\mathcal{B}$, the energy density $\mathcal{E}$, the potential $V$, and the vorticities $\mathcal{Q}_{1,2}$ in $\phi_{1,2}$ (with red and blue, respectively). The color scheme used for the first 3 columns is described in the text. Each row corresponds to a different value of the potential parameter $M^{2}$.

Definition 1. Vortex links are degenerate, if they possess a mathematical junction which makes the counting of the linking number impossible.

For a detailed discussion of the linking number, see Ref. [2]. In particular, the vortex links are not necessarily degenerate, if at a given level set, the isosurfaces merely touch each other. That is, if the vortices do not touch each other by increasing the level-set value from $1 / 2$ to a higher 


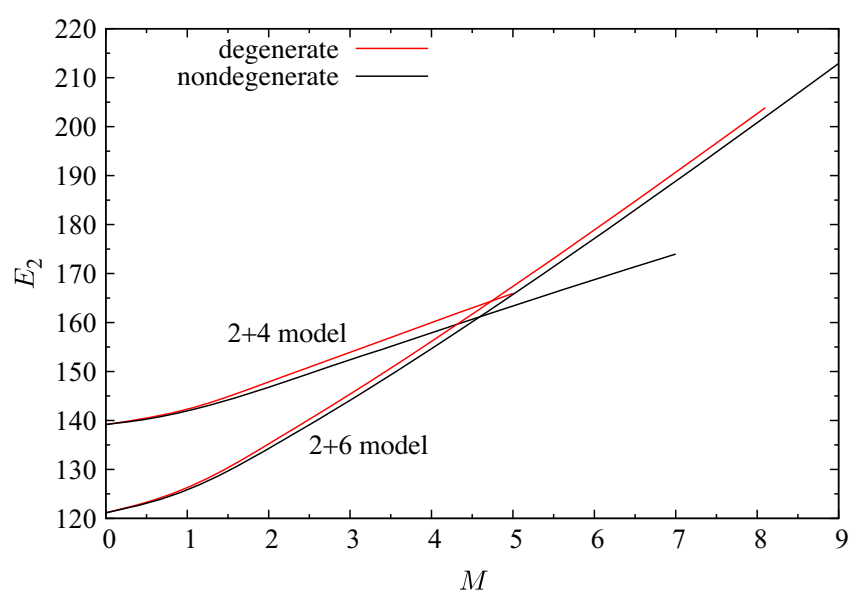

FIG. 4. Energy of degenerate versus nondegenerate $B=2$ Skyrmion solutions for both the $2+4$ model and the $2+6$ model.

value of the maximum density, then they are not degenerate by definition 1 .

According to definition 1 , the $B=2$ Skyrmion shown in Fig. 2 is degenerate until a sufficiently high value of the potential parameter is reached: i.e., at $M \sim 5$ for the $2+4$ model and $M \sim 9$ for the $2+6$ model the degeneracy is broken spontaneously and the vortex links fall into a nondegenerate state where the linking number is clearly two- this can be seen from the potential (3rd column) and the vorticities (4th column) of the figure. For the $2+4$ model, the baryon charge and the total energy densities are only slightly deformed, whereas for the $2+6$ model once the degeneracy is broken, the baryon charge shows clear signs of the linked vortices inside the Skyrmion and the energy density takes the same shape as the potential-i.e., as two doubly linked vortices.

One could now expect that the degenerate soliton becomes unstable for a sufficiently large $M$ and decays into the nondegenerate state. This begs the question, what is the phase diagram of these two states as function of $M$. In particular, which is the stable state for smaller values of $M$. In order to investigate this, we will use the $B=2$ Skyrmion with broken degeneracy [see the last lines of Figs. 2(a) and 2(b)] as the initial guess and decrease $M$ to zero-and while doing so, we calculate the total energy. The result is shown in Fig. 3 and for both models, the linked vortices are nondegenerate all the way as $M$ tends to zero, thus allowing one to count the topological degree as the linking number of the two vortex species, see Ref. [2]. In order to conclude which of the two solutions is the stable one, we plot the energies in Fig. 4. Indeed, the solutions with nondegenerate vortices turn out to be the stable ones. For the stable solutions, the linked vortices are visible in the energy density from $M \gtrsim 3$ in the $2+6$ model, whereas for the $2+4$ model they are only barely visible at $M=6$.

One could posit that starting with the (transformed) rational map approximated Skyrmion is the reason for (a)
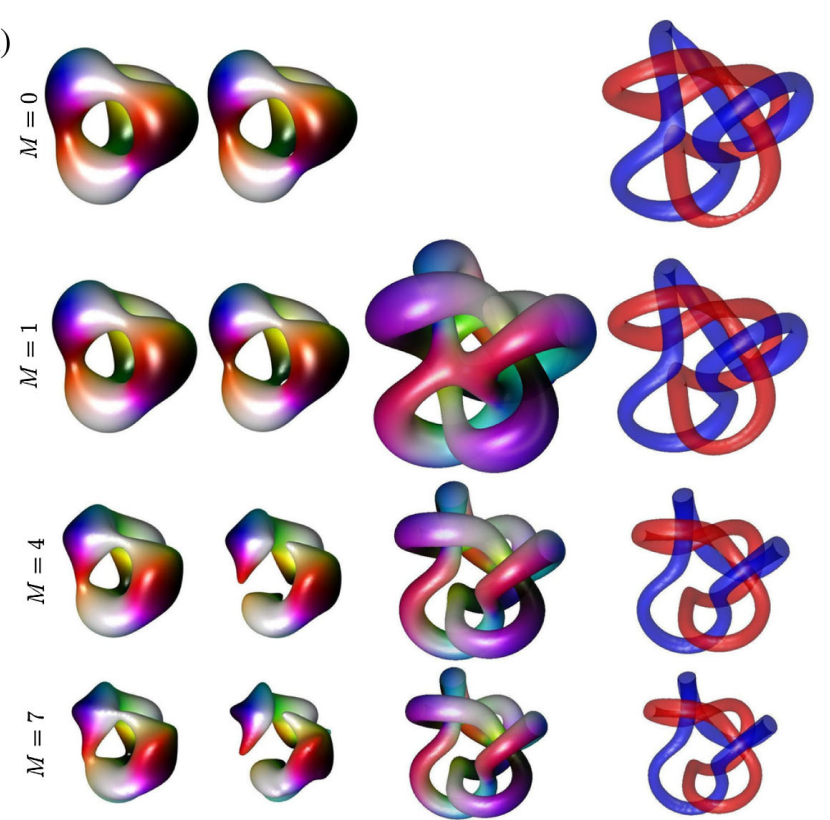

(b)
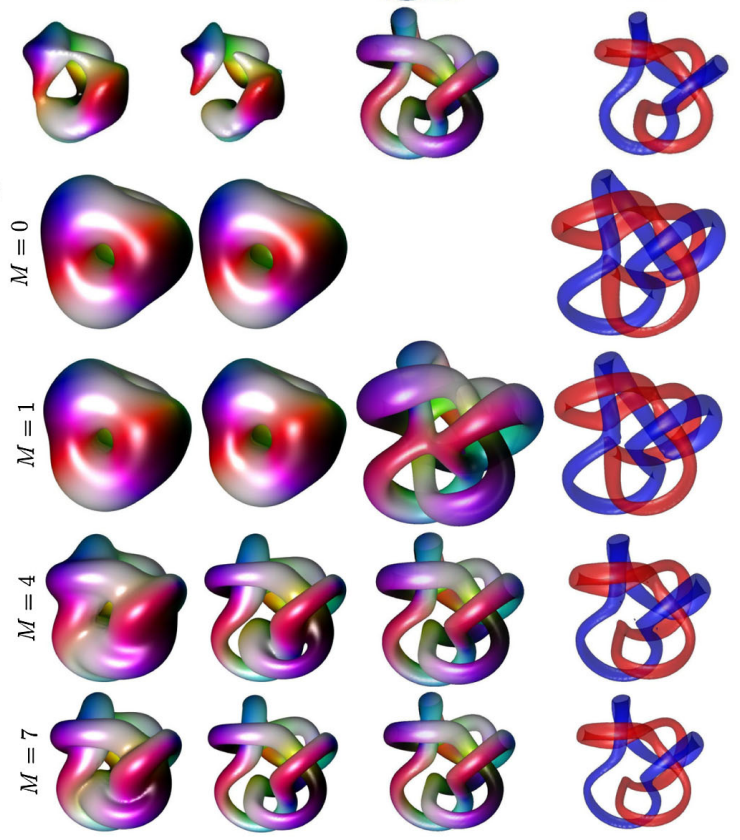

FIG. 5. The $B=3$ Skyrmion in the miscible BEC-Skyrme (a) $2+4$ model (b) $2+6$ model. The four columns show isosurfaces of: the topological baryon charge density $\mathcal{B}$, the energy density $\mathcal{E}$, the potential $V$, and the vorticities $\mathcal{Q}_{1,2}$ in $\phi_{1,2}$ (with red and blue, respectively). The color scheme used for the first 3 columns is described in the text. Each row corresponds to a different value of the potential parameter $M^{2}$.

ending up in the metastable state. Hence, we have performed a scattering of two $B=1$ Skyrmions in the attractive channel. The initial state was prepared by means of the asymmetric product Ansatz of two 1-Skyrmions, which was then transformed into the frame compatible with this model's vacuum using Eq. (30). Surprisingly, the result for $M=1$ was the metastable "degenerate" state instead of the stable ground state.

We will now consider the $B=3$ topological sector, for which the results are shown in Fig. 5. The $M=0$ row of Fig. 5(a) [Fig. 5(b)] shows the normal tetrahedrally 

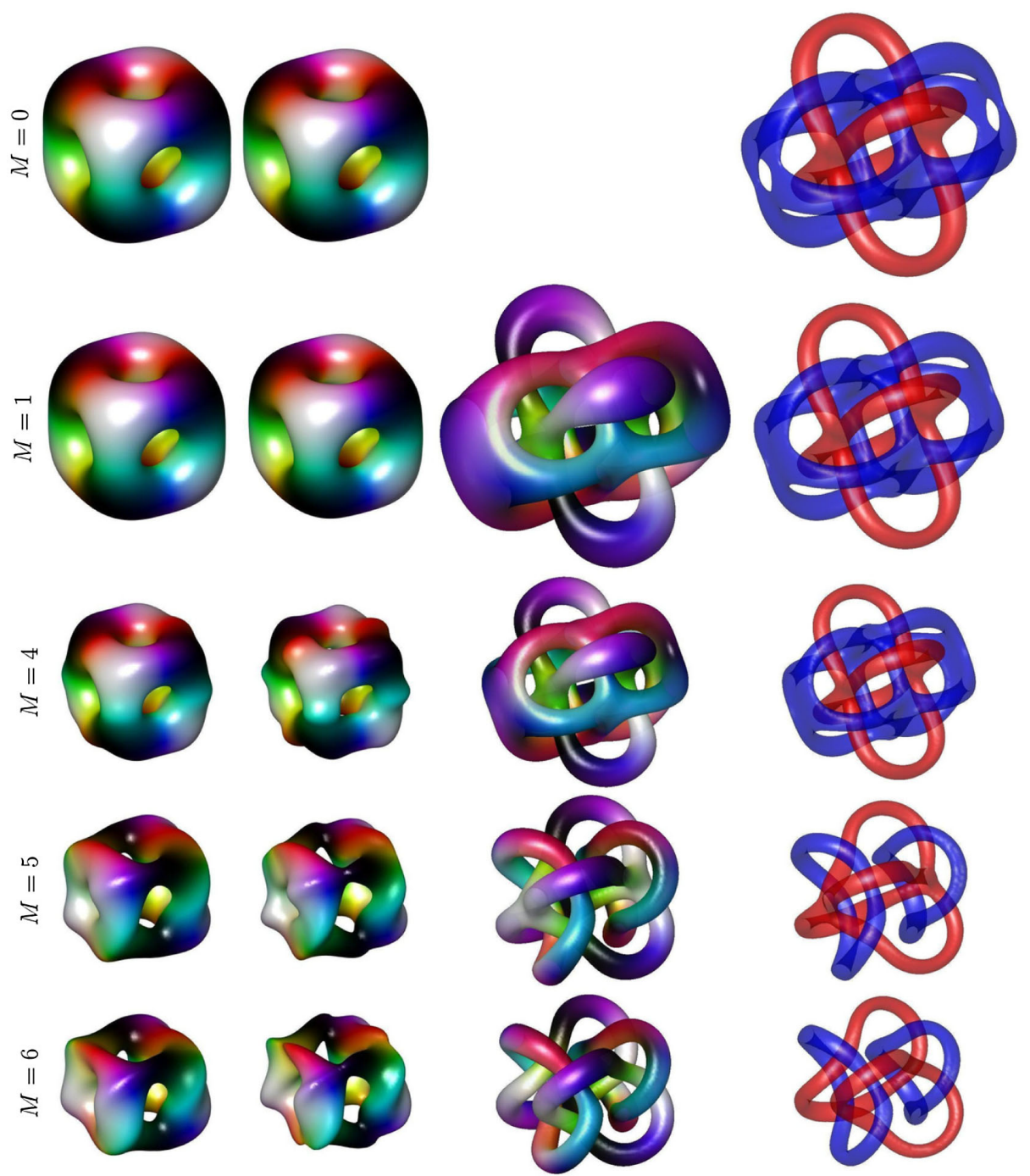

FIG. 6. The metastable $B=4$ Skyrmion in the miscible BEC-Skyrme $2+4$ model. The four columns show isosurfaces of: the topological baryon charge density $\mathcal{B}$, the energy density $\mathcal{E}$, the potential $V$, and the vorticities $\mathcal{Q}_{1,2}$ in $\phi_{1,2}$ (with red and blue, respectively). The color scheme used for the first 3 columns is described in the text. Each row corresponds to a different value of the potential parameter $M^{2}$.

symmetric Skyrmion in the $2+4(2+6)$ model. The triply linked vortex lines are visible in the 4 th column of the figure and they are not degenerate for this soliton solution. Turning on a finite $M$ yields the potential energy (3rd column) in a similar shape as the vorticities (4th column). For $M=1$ it may look like the potential (3rd column) shows signs of degeneracy, but this is merely an illusion due to the level set and is not a degeneracy according to definition 1 ; this can be seen by inspecting the vorticities in the fourth column of the figure. In the $2+4$ model, the energy density isosurface changes for large values of $M$ (i.e., $M \sim 4-7$ ), but instead of becoming similar to the shape of the potential isosurface ( 3 rd column) it separates into four lumps centered at the corners of the tetrahedron with hints of the vortex lines sticking out. In contrast, for the $2+6$ model, the energy density isosurface becomes very similar in shape to that of the potential energy for large values of $M$ (i.e., $M \sim 4-7$ ), see the second column of Fig. 5. This can be traced back to the perfect fluid properties of the BPS-Skyrme term [36].

We now turn to the $B=4$ sector, where the Skyrmion solution for $M=0$ is octahedrally symmetric (which is the dual symmetry of the cube). First we obtain solutions for various values of $M$ using the initial data which are transformed rational map solutions for the $2+4$ model in Fig. 6 and for the $2+6$ model in Fig. 7. In both the $2+4$ and the $2+6$ model, the solution obtained for small values of $M$ is degenerate according to definition 1, see the $3 \mathrm{rd}$ and 4th columns of Figs. 6 and 7, respectively. The critical value of the mass parameter is $M_{\text {crit }} \sim 4.3$ for the $2+4$ model and $M_{\text {crit }} \sim 6$ for the $2+6$ model. Interestingly, the breaking of degeneracy that happens at this value of $M$ is 

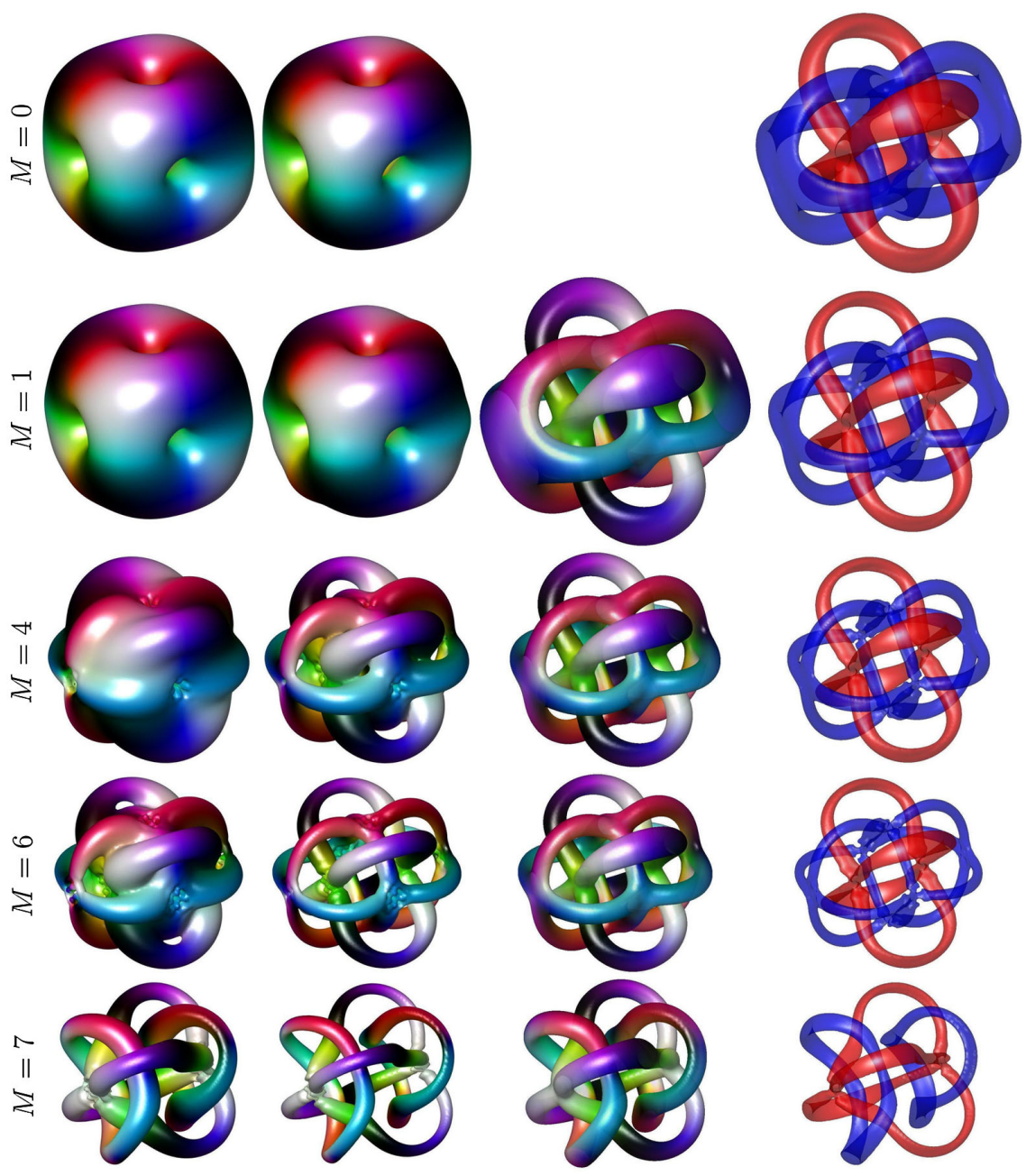

FIG. 7. The metastable $B=4$ Skyrmion in the miscible BEC-Skyrme $2+6$ model. The four columns show isosurfaces of: the topological baryon charge density $\mathcal{B}$, the energy density $\mathcal{E}$, the potential $V$, and the vorticities $\mathcal{Q}_{1,2}$ in $\phi_{1,2}$ (with red and blue, respectively). The color scheme used for the first 3 columns is described in the text. Each row corresponds to a different value of the potential parameter $M^{2}$.

only partial. That is, the degeneracy of the blue vortices breaks spontaneously, but that of the red vortices sustains, see the fourth column in Figs. 6 and 7. The lower-in-energy metastable state, with only the red vortices being degenerate, can be seen for $M=5$ in Fig. 6 and for $M=7$ in Fig. 7. If we continue to increase the mass $M$, we expect to be able to break the lower-in-energy metastable state with only the red vortices being degenerate, and indeed that happens at $M_{\text {crit }}^{\prime} \sim 5.4$ in the $2+4$ model. For the $2+6$ model, we have not been able to find this critical value of the mass parameter; by increasing $M$ to $M=15$, the lowerin-energy metastable state is still metastable. Beyond that value of the mass parameter, we do not trust the accuracy of the code for the lattices used in these simulations.

Figure 8 shows the lower-in-energy metastable states, where only the red vortices are degenerate while the blue vortices are nondegenerate, see the fourth column of the figure. Figure 9 shows the stable nondegenerate soliton solution, which is nondegenerate in both the red $\left(\phi_{1}\right)$ and blue $\left(\phi_{2}\right)$ vortices. In order to back up our claim, we calculate the energies for the different metastable and the stable solutions as functions of $M$ and the result is shown in Fig. 10. It is clear both from the energies in Fig. 10 and from inspecting the baryon charge (1st column) and energy ( 2 nd column) isosurfaces of Figs. 6-9, that the $M=0$ solution is energetically and physically the same solution. Nevertheless, the vorticities plots are clearly different in Figs. 6-7 versus Fig. 8 versus Fig. 9. This is because, for vanishing potential, the difference is merely a rotation of the 2-sphere in the language of Ref. [2], which is a subgroup of $\mathrm{O}(4)$ and hence does not change the energy or the physics of the Skyrmion with massless pions. Once, the potential (7) is turned on, this rotation is no longer a symmetry and the potential picks out the nondegenerate vortex links as the ground state. Perhaps surprisingly, there are metastable states with residual 

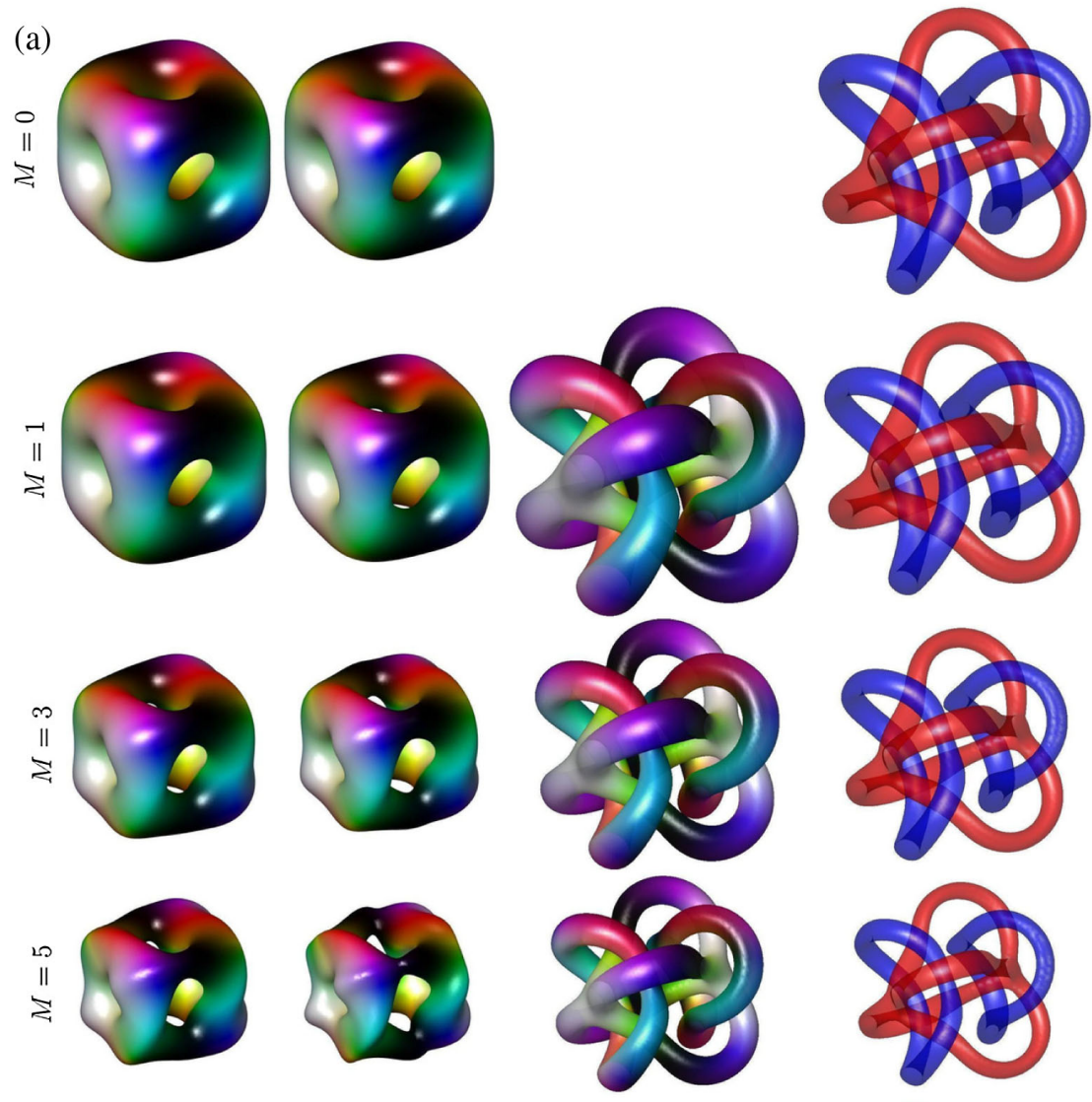

(b)
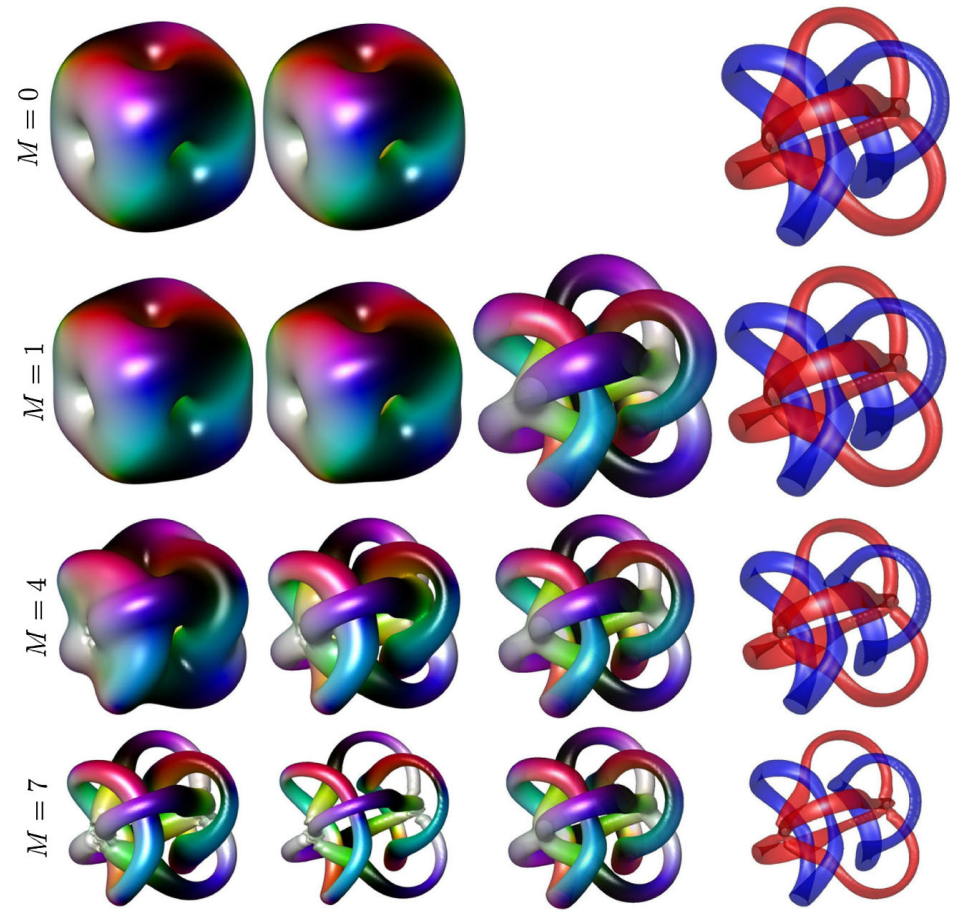

FIG. 8. The lower-in-energy metastable $B=4$ Skyrmion in the miscible BEC-Skyrme (a) $2+4$ model (b) $2+6$ model. The four columns show isosurfaces of: the topological baryon charge density $\mathcal{B}$, the energy density $\mathcal{E}$, the potential $V$, and the vorticities $\mathcal{Q}_{1,2}$ in $\phi_{1,2}$ (with red and blue, respectively). The color scheme used for the first 3 columns is described in the text. Each row corresponds to a different value of the potential parameter $M^{2}$. 
(a)
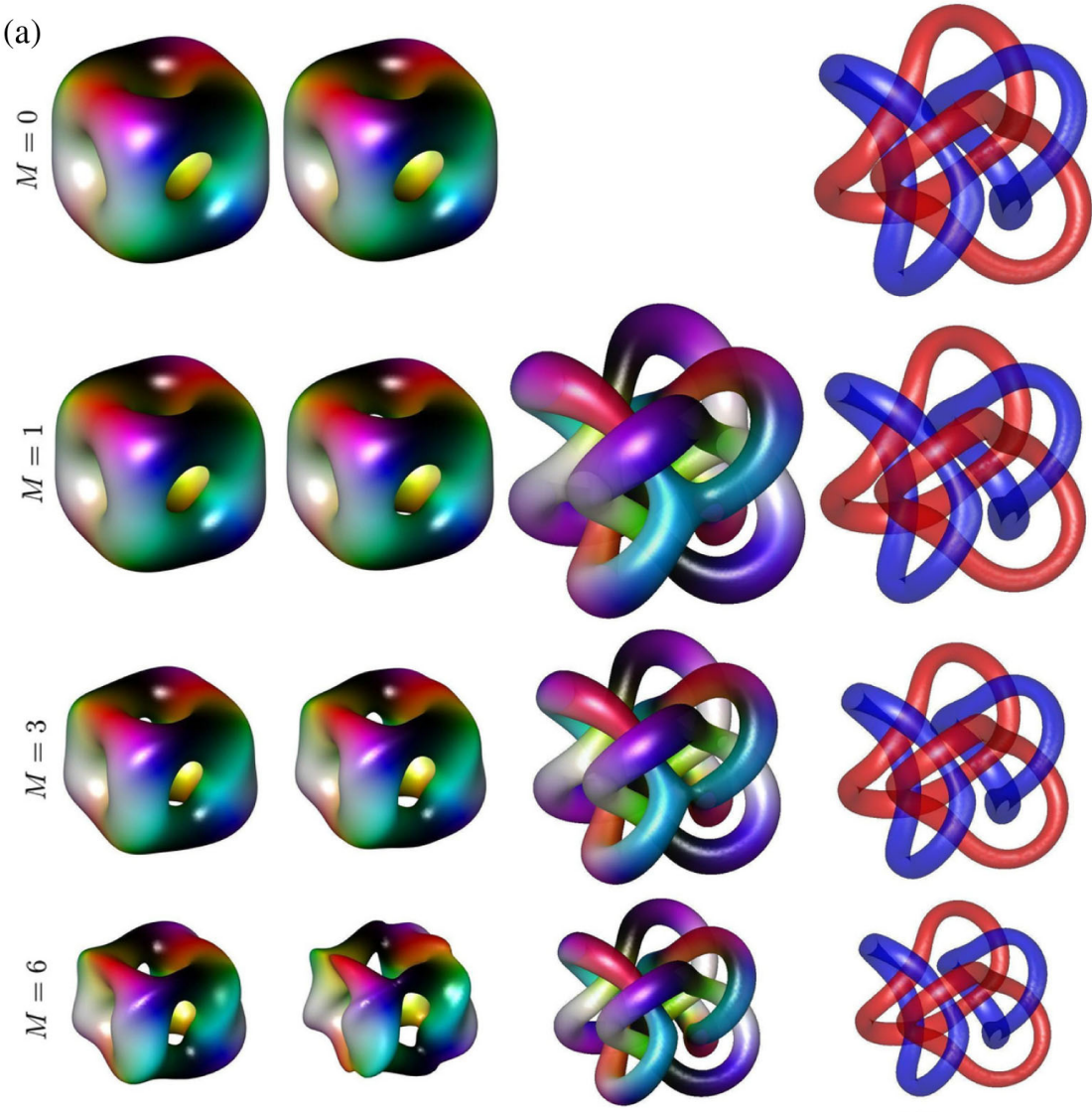

(b)
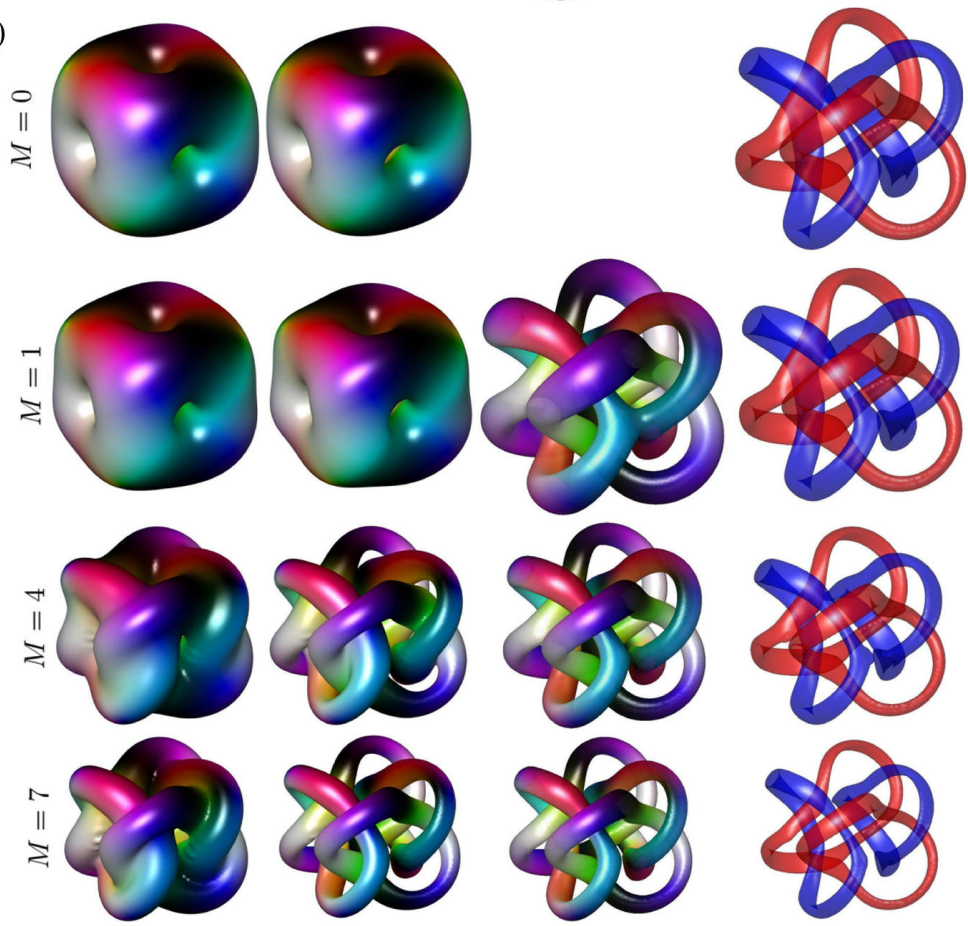

FIG. 9. The stable $B=4$ Skyrmion in the miscible BEC-Skyrme (a) $2+4$ model (b) $2+6$ model. The four columns show isosurfaces of: the topological baryon charge density $\mathcal{B}$, the energy density $\mathcal{E}$, the potential $V$, and the vorticities $\mathcal{Q}_{1,2}$ in $\phi_{1,2}$ (with red and blue, respectively). The color scheme used for the first 3 columns is described in the text. Each row corresponds to a different value of the potential parameter $M^{2}$. 


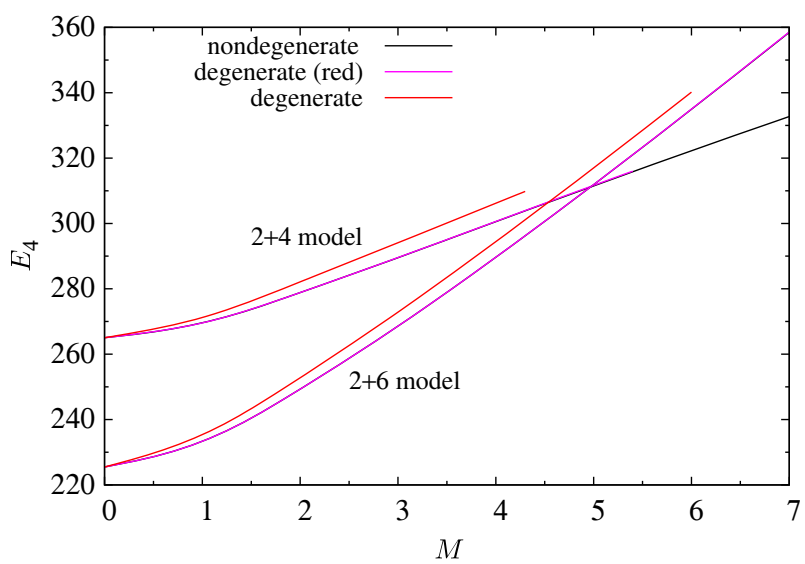

(a)

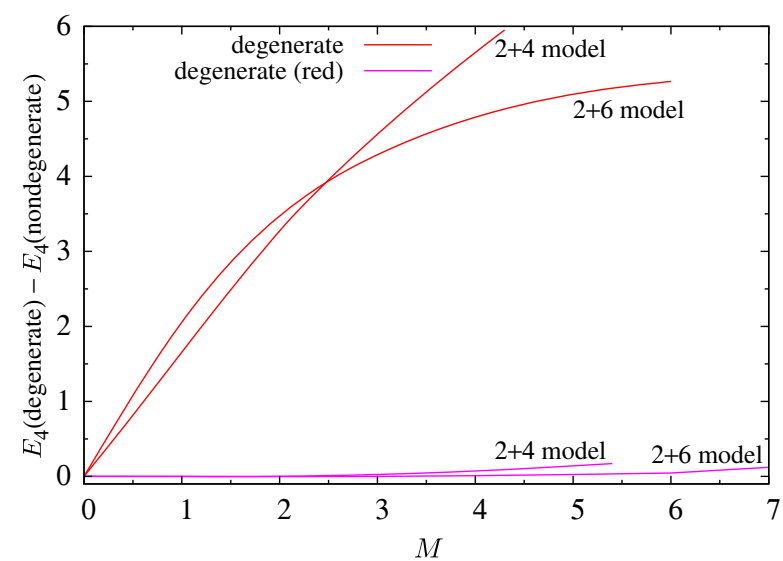

(b)

FIG. 10. (a) Energy of degenerate versus nondegenerate $B=4$ Skyrmion solutions for both the $2+4$ model and the $2+6$ model. There is a degenerate state with almost the same energy as the nondegenerate state, where only the red vortex $\left(\phi_{1}\right)$ is degenerate, but the blue vortex $\left(\phi_{2}\right)$ is nondegenerate. (b) The energy difference between the degenerate states and the nondegenerate "ground state."

degeneracy or even partial residual degeneracy left. This was not expected.

Although the fully degenerate state shown in Figs. 6 and 7 is clearly heavier on the energy graph 10 (a) than the other two solutions, the difference between the lower-inenergy metastable state of Fig. 8 and the stable nondegenerate solution of Fig. 9 is actually so small, that we have plotted the difference between the energies of the degenerate and the nondegenerate states in Fig. 10(b). Clearly the partially degenerate solution with only the red vortices being degenerate, has almost the same energy as the nondegenerate stable solution. This was not expected in the model with the potential (7) turned on.

We will now summarize the numerical results so far. The $B=1$ case does not possess degeneracy in any frame, see Ref. [2], but the second vortex is not necessarily physical, as it can go off to infinity as a "vacuum" vortex. For $B=2$ the standard frame after the transformation (30) turns out to give a degenerate solution. Cranking up the potential parameter turns this metastable degenerate state into an unstable state and the true nondegenerate "ground state" is found. For $B=3$ the found solution does not possess degeneracy. For $B=4$ the standard frame after rotation yields a metastable state with degeneracy which is higher in energy than another metastable state with partial degeneracy. The stable solution, however, in all cases is the nondegenerate one.

Since we have shown the first four Skyrmion solutions in great detail, we will only depict the stable ones of the next four Skyrmions $(B=5$ through $B=8)$ and only for $M=0,5$ : For the $2+4(2+6)$ model the results are shown in Fig. 11 (Fig. 12). The stable Skyrmions without the potential (i.e., with $M=0$ ) have dihedral $D_{2 d}$, dihedral $D_{4 d}$, icosahedral and dihedral $D_{6 d}$ symmetry, respectively.

It turns out that for $B=5$, there is a stable solution which possesses nondegenerate vortex links, see Figs. 11(a) and 12(a), as well as an unstable solution with degenerate links (not shown). The energies of the two branches of solutions are shown in Fig. 15(a) and the situation is very similar to that of the $B=2$ Skyrmion.

For the $B=6,7$ Skyrmions, we only find a stable solution shown in Figs. 11(b), 11(c), 12(b), and 12(c) for the $2+4$ model and the $2+6$ model, respectively. Whereas the $B=7$ solution is clearly nondegenerate, the $B=6$ solution looks almost degenerate, see the fourth column of the figures for $M=0$. This turns out to be an artifact of the level set and indeed the degeneracy according to definition 1 is not present.

The last Skyrmion solution is the $B=8$ solution, for which the stable Skyrmion in this model is shown in Figs. 11(d) and 12(d) for the $2+4$ model and the $2+6$ model, respectively. For all the Skyrmion solutions in the $2+4$ model, see Fig. 11, the vortex links are less visible than in the $2+6$ model, see Fig. 12, even for large $M$. That being said, the deformations of the Skyrmion solutions in the $2+4$ model are definitely visible for large values of $M$, but in the $2+6$ model, the shape of the energy density (2nd column) converges quickly to that of the potential (3rd column), which by comparison to the vorticities (4th column) represents the two flavors of vortex, which when nondegenerate are linked exactly $B$ times [2].

The story for the first seven baryon numbers (one through seven) was that the solutions with degenerate vortices had higher energies than solutions with nondegenerate vortices. For the $B=8$ Skyrmion it turns out to be more complicated. First of all, there exists both a fullerenelike solution, which is predicted by the rational map approximation [35] as well as a solution that is made up of two cubes attached to each other [7]. In the Skyrme model with a standard pion mass there are in fact two solutions, which are made of two cubes ( $B=4$ solutions) next to each other: One is a translation of the first, whereas 
(a)
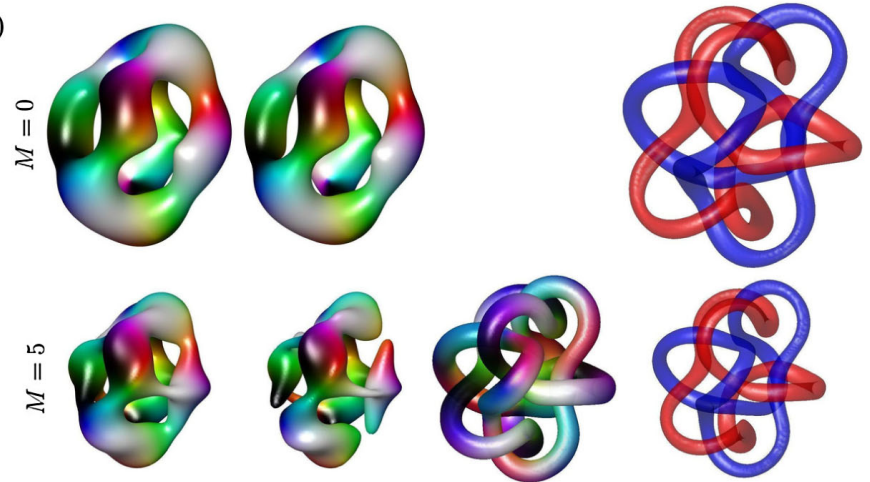

(b)
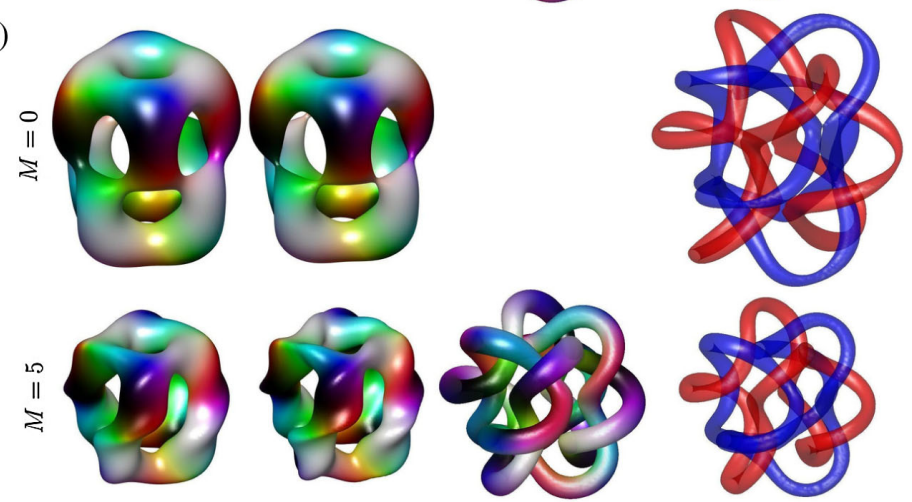

(c)

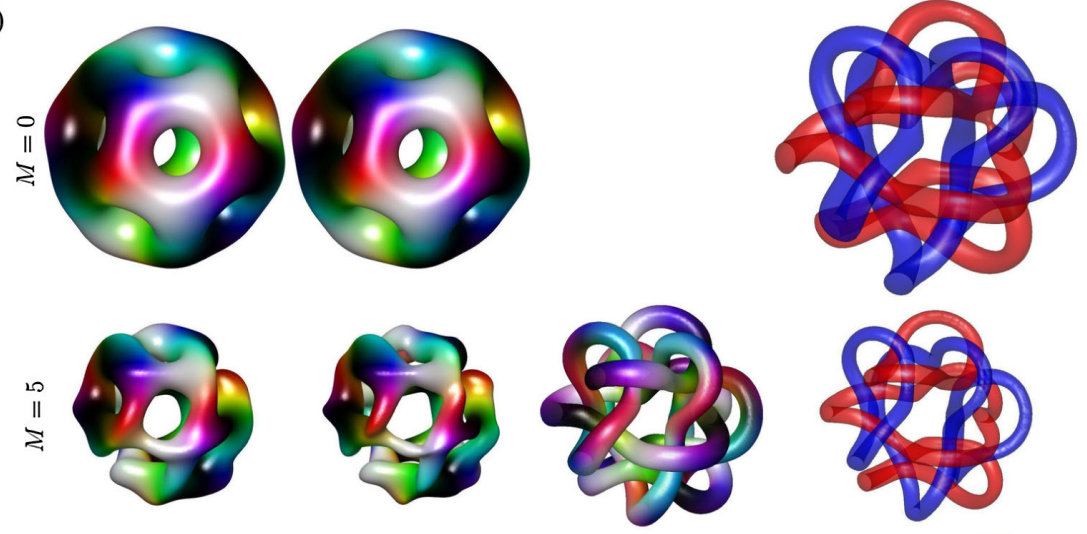

(d)

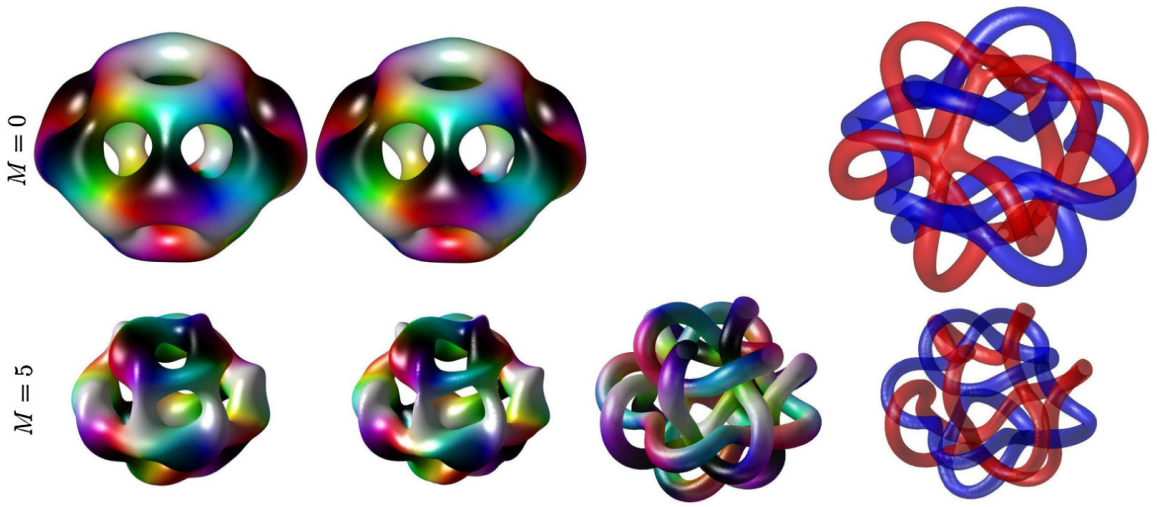

FIG. 11. The stable (a) $B=5$, (b) $B=6$, (c) $B=7$, (d) $B=8$ Skyrmions in the miscible BEC-Skyrme $2+4$ model. 
(a)

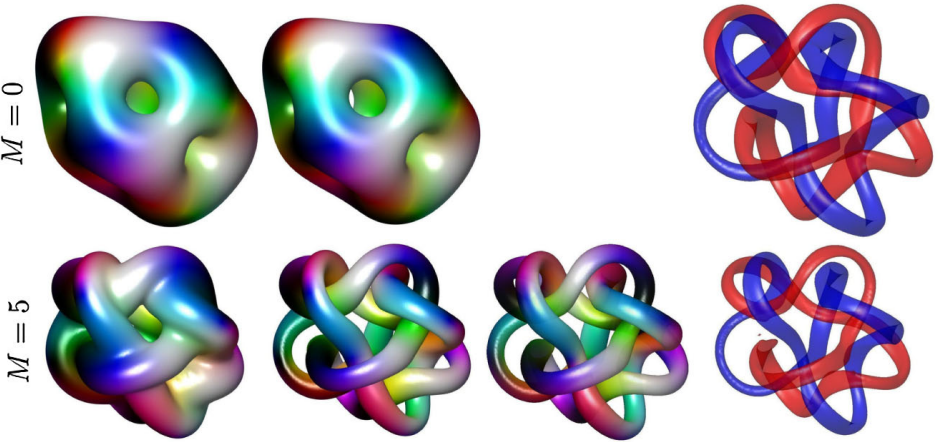

(b)
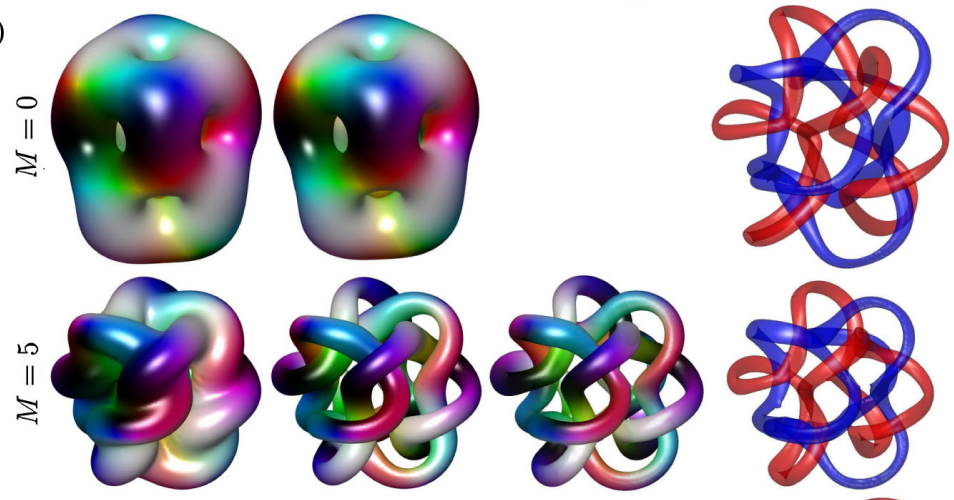

(c)
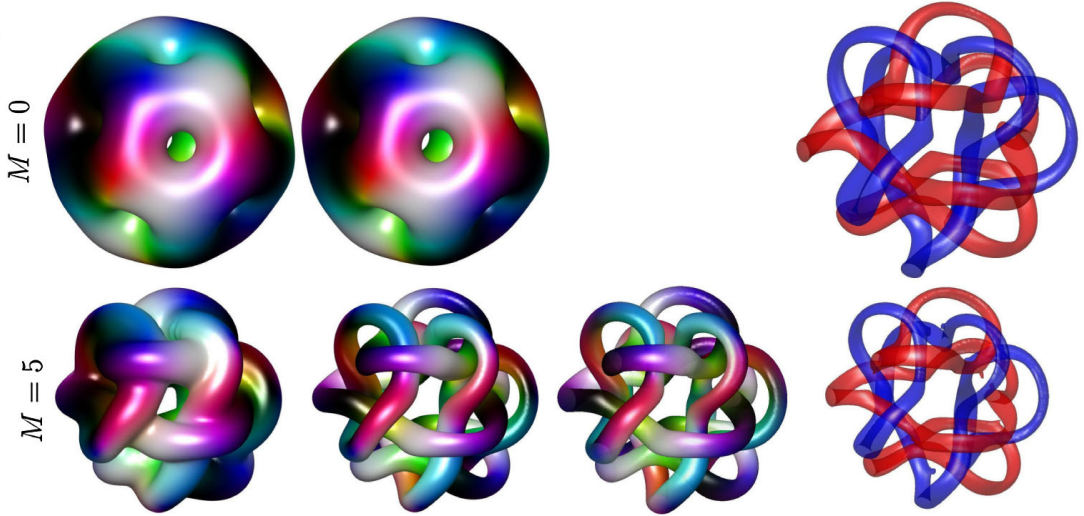

(d)
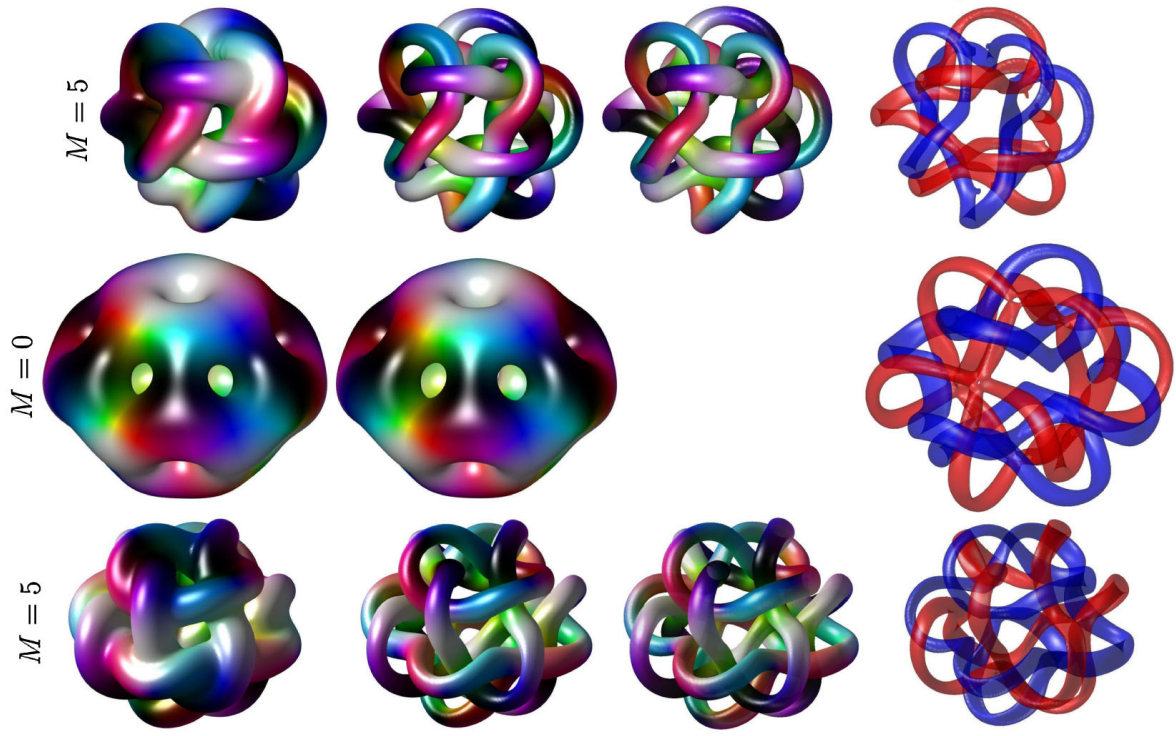

FIG. 12. The stable (a) $B=5$, (b) $B=6$, (c) $B=7$, (d) $B=8$ Skyrmions in the miscible BEC-Skyrme $2+6$ model. The four columns show isosurfaces of: the topological baryon charge density $\mathcal{B}$, the energy density $\mathcal{E}$, the potential $V$, and the vorticities $\mathcal{Q}_{1,2}$ in $\phi_{1,2}$ (with red and blue, respectively). The color scheme used for the first 3 columns is described in the text. Each row corresponds to a different value of the potential parameter $M^{2}$. 

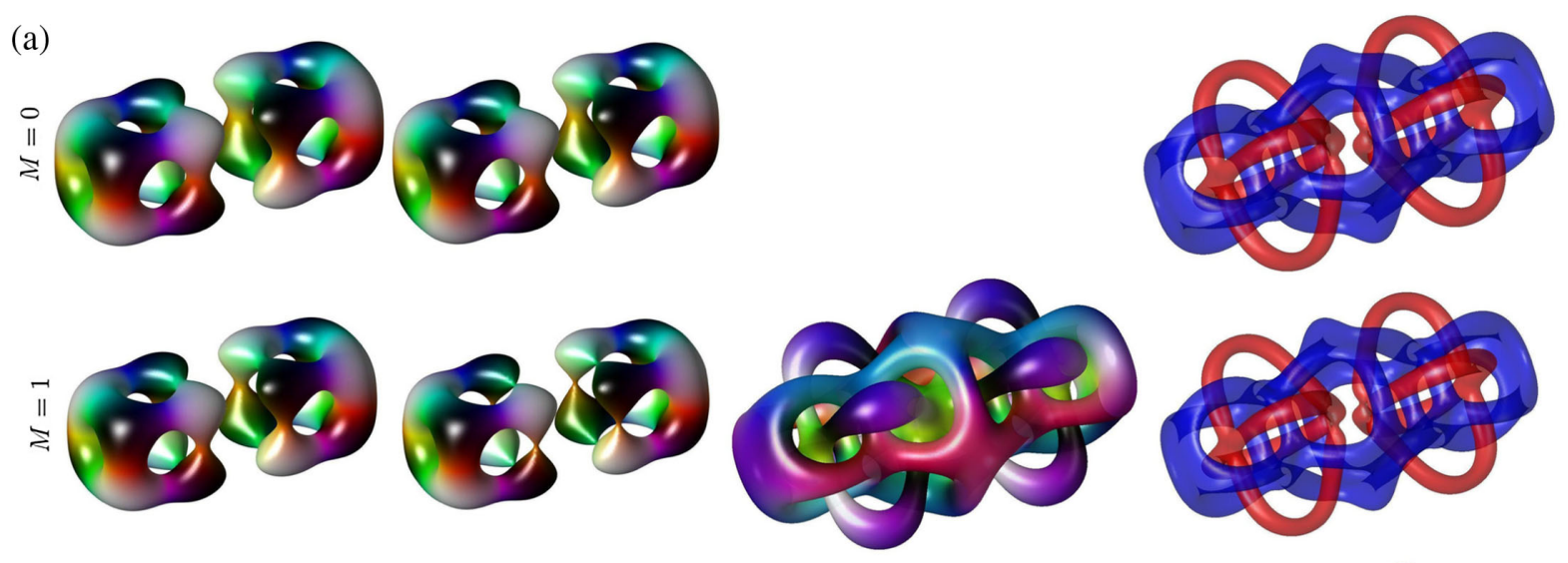

(b)
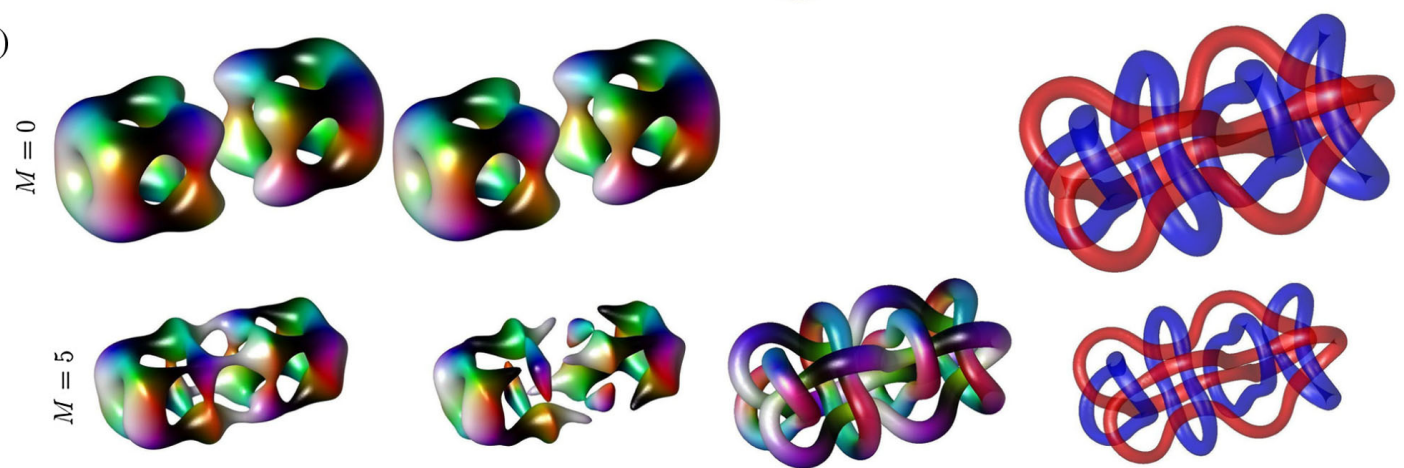

(c)
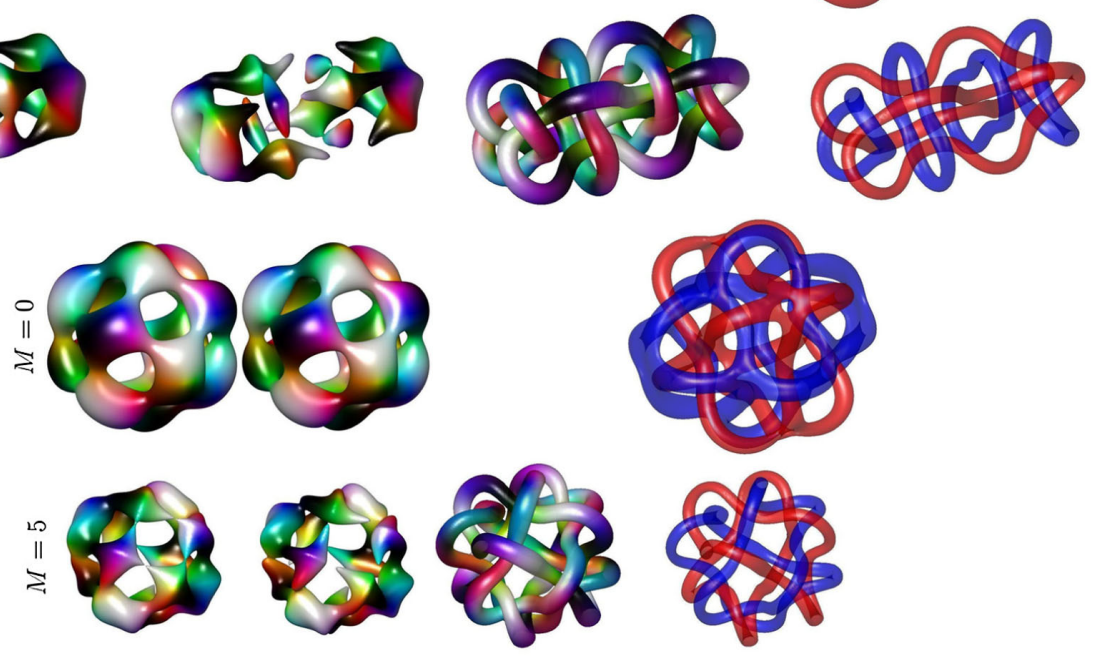

FIG. 13. The metastable $B=8$ Skyrmions in the miscible BEC-Skyrme $2+4$ model. The three metastable solutions are: (a) two degenerate cubes, (b) two nondegenerate cubes, and (c) a fully degenerate fullerene-like solution. The four columns show isosurfaces of: the topological baryon charge density $\mathcal{B}$, the energy density $\mathcal{E}$, the potential $V$, and the vorticities $\mathcal{Q}_{1,2}$ in $\phi_{1,2}$ (with red and blue, respectively). The color scheme used for the first 3 columns is described in the text. Each row corresponds to a different value of the potential parameter $M^{2}$.

in the second solution the translated copy is rotated by 90 degrees around the axis joining them. Both of these $B=8$ Skyrmions have lower energy than the fullerene-like dihedrally symmetric solution, once a pion mass is turned on [7] and the critical value for the mass turns out to be rather small. In this model, on the other hand, the solutions with the two cubes are not lower in energy for any value of the potential parameter $M$. Furthermore, only the first version of the two cubes exist; that is, the one where the $B=4$ solutions are translated copies of each other, see Figs. 13(a), 13(b), 14(a), and 14(b). By that, we mean that the twisted solution where one of the cubes is rotated by 90 degrees around the axis joining them, does not exist in this model as it collapses into a fullerene-like solution, see Figs. 13(c) and 14(c), albeit with higher energy than the original $D_{6 d}$ symmetric solution predicted by the rational map approximation - this is because this fullerene-like solution is fully degenerate at small $M$. This collapse of the twisted double cube $B=8$ Skyrmion has been observed also in other Skyrme-like models, see Ref. [34]. The existence of another fullerene-like solution has also been observed in Ref. [34]. Of course, such existence or nonexistence of the different solutions and which one is the global minimizer of the energy functional, is very dependent on the model and in particular on the potential. 
(a)
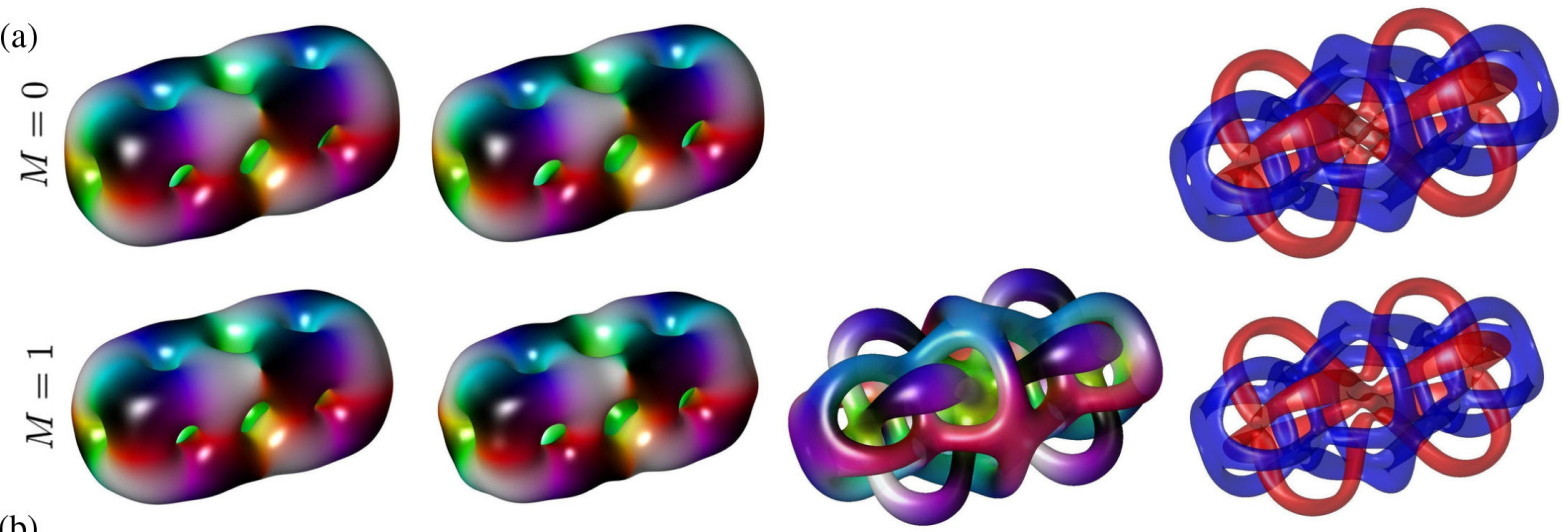

(b)
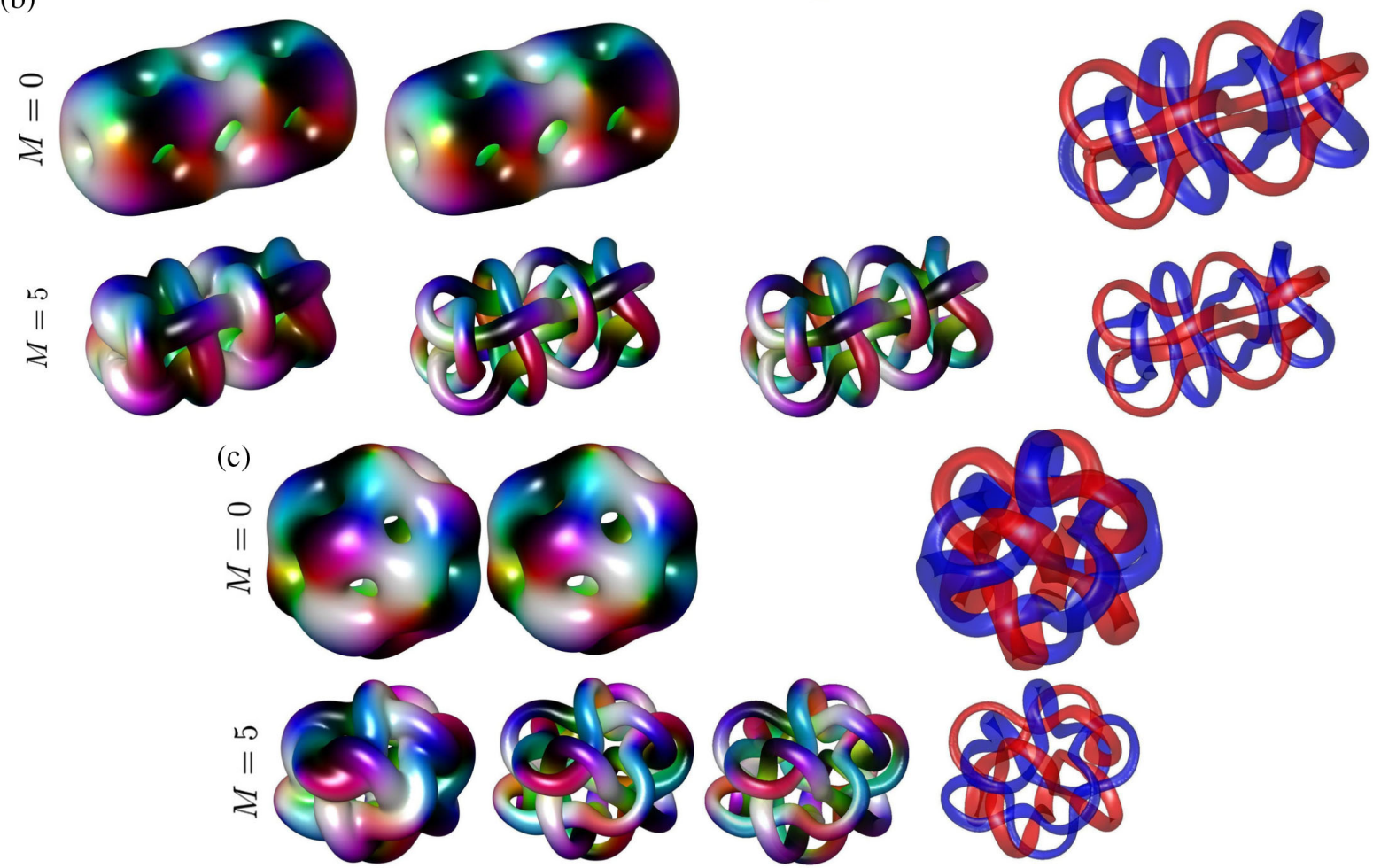

FIG. 14. The metastable $B=8$ Skyrmions in the miscible BEC-Skyrme $2+6$ model. The three metastable solutions are: (a) two degenerate cubes, (b) two nondegenerate cubes, and (c) a fully degenerate fullerene-like solution. The four columns show isosurfaces of: the topological baryon charge density $\mathcal{B}$, the energy density $\mathcal{E}$, the potential $V$, and the vorticities $\mathcal{Q}_{1,2}$ in $\phi_{1,2}$ (with red and blue, respectively). The color scheme used for the first 3 columns is described in the text. Each row corresponds to a different value of the potential parameter $M^{2}$.

The energies of the different $B=8$ solutions are shown in Figs. 15(b)-(d). It turns out that the $D_{6 d}$ dihedrally symmetric (which is the symmetry only at $M=0$ ) fullerene-like solution is the global minimizer of the energy functional for all values of $M$ studied in this paper-even though it is partially degenerate. The lowestlying metastable state is another fullerene like structure, which is fully degenerate. The highest-energy solutions are given by two cubes joined together. It turns out there are indeed two different $B=8$ solutions made of cubes, but the difference is not whether they are rotated with respect to each other or not. The difference between them is whether the vorticities are degenerate or not. As one could guess by now, the one with degenerate vorticities has the highest energy and is unstable above $M \sim 2.1$ for both the $2+4$ and the $2+6$ models, see Figs. 13(a) and 14(a). A lower-lying $B=8$ solution made of $B=4$ cubes has nondegenerate vorticities and is metastable for all the values of $M$ studied, see Figs. 13(b) and 14(b). Although this solution may look degenerate, it is not according to definition 1 ; the vorticities are just going very close to one another for the red vortices $\left(\phi_{1}\right)$. The mystery remains, however, that the stable solution seems to have partially degenerate vortices. 


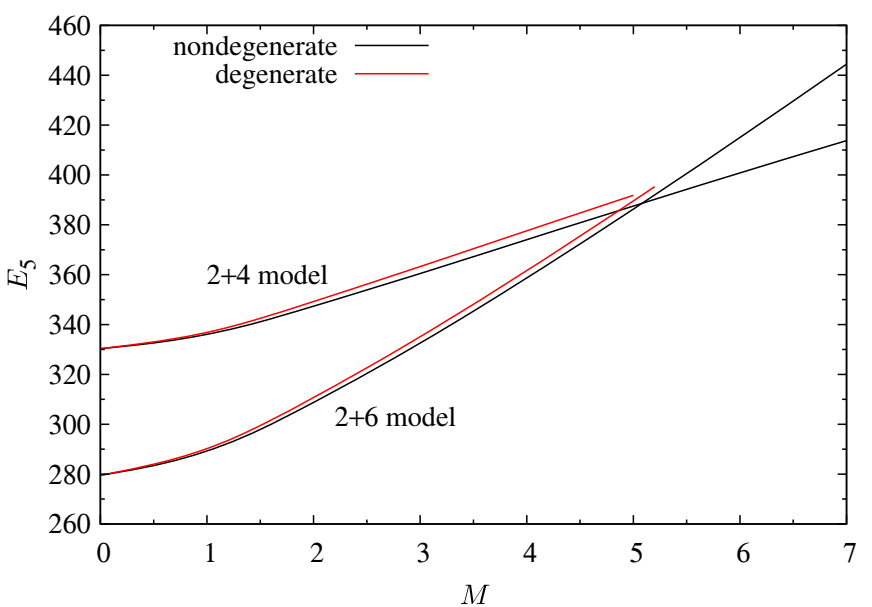

(a) $B=5$

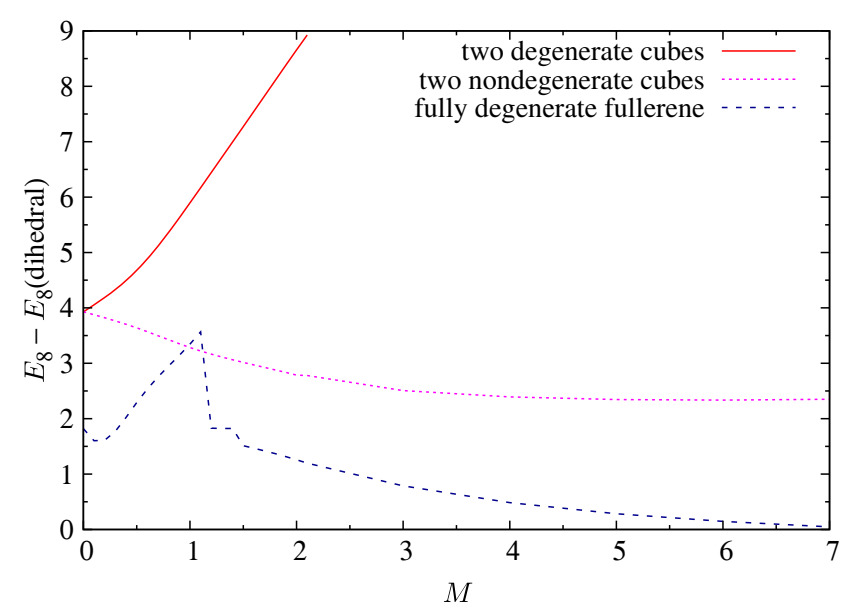

(c) $B=8: 2+4$ model

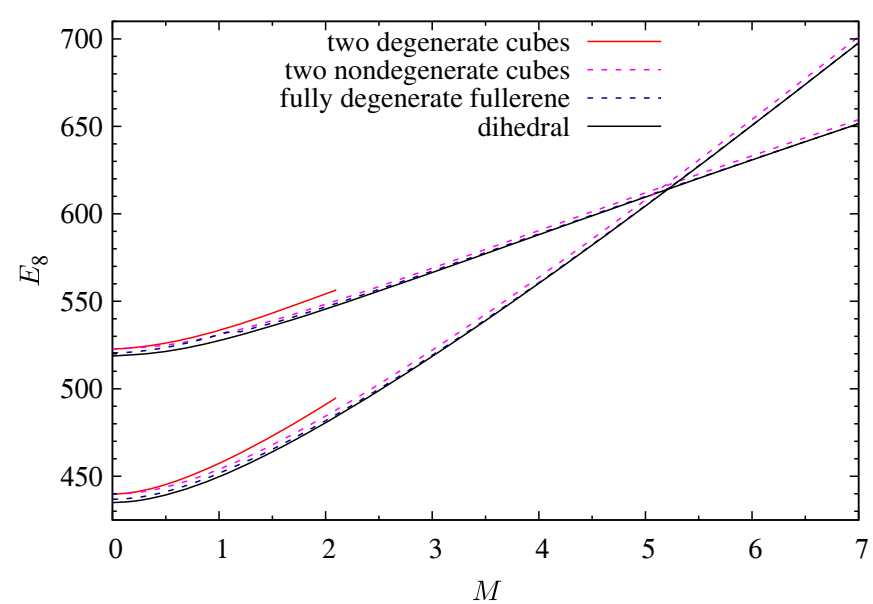

(b) $B=8$

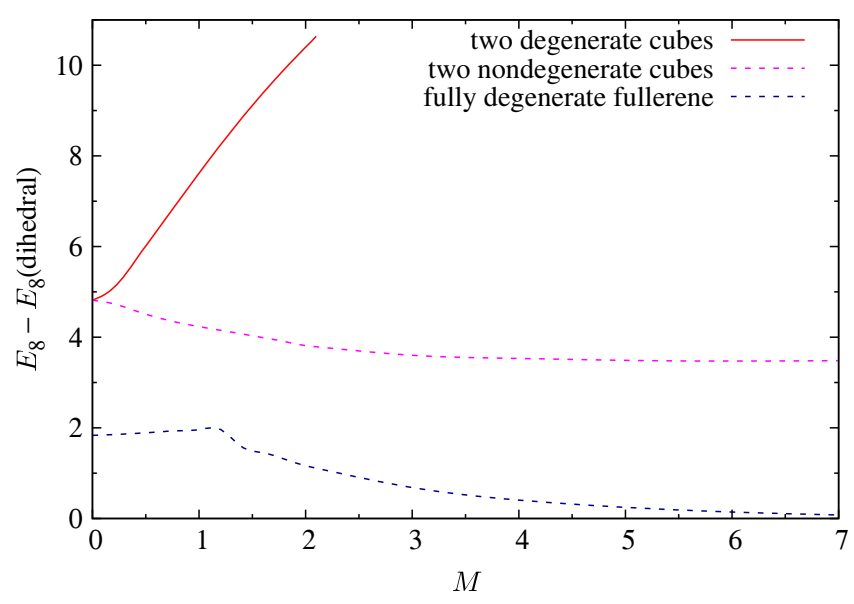

(d) $B=8: 2+6$ model

FIG. 15. Energy of degenerate versus nondegenerate (a) $B=5$ and (b) $B=8$ Skyrmion solutions for both the $2+4$ model and the $2+6$ model. A difference plot between the stable "dihedral" solution and the other metastable solutions for (c) the $2+4$ model and (d) the $2+6$ model.

\section{DISCUSSION}

In this paper, we have considered the miscible BECSkyrme model, which is the generalized Skyrme model with fourth-order and sixth-order derivative terms augmented by the BEC-inspired potential, but with the opposite overall sign of the potential compared with the previously considered immiscible BEC-Skyrme model. The symmetries of the Lagrangian are unchanged, but the vacuum state is now connected and hence the continuous symmetry is completely broken. The interesting point is that, unlike the immiscible BEC Skyrme model, this model possesses two physical vortex strings: one in $\phi_{1}$ and another in $\phi_{2}$, with $\phi_{1,2}$ being the two complex fields of the (nonlinear sigma) model. These two vortex lines have been proven in Ref. [2] to have linking number $Q=B$ equal to the baryon number, i.e., the topological degree of the Skyrmion, under the condition that a certain projection is regular. It so happens that choosing a standard frame for the Skyrmion often yields a situation which is not regular, and the vortex lines degenerate-thus making it impossible to define the linking number (this has no consequence for the definition of the baryon number). The potential at hand in this model makes such a degeneracy energetically unfavorable and hence for large enough potential parameter, $M$, the model naturally possesses Skyrmion solutions which are made of nondegenerate vortex lines that are linked exactly $B$ times, with $B$ being the topological degree of the Skyrmions.

The general lesson learned is that if both flavors of vortex (i.e., both the red and the blue vortices) are degenerate, then the solution is a metastable state. A lower energy solution can be found where the degeneracy is broken in one or both flavors of vortex. We can thus make the following conjecture: 
Conjecture 1. The lowest-energy Skyrmion solution in the miscible BEC-Skyrme model has nondegenerate vortices in both vortex species for all values of $M$ and for all topological degrees $B$.

The conjecture is verified by the calculations in the topological sectors $B=1$ through $B=7$ in this paper.

A curious exception seems to occur for the $B=8$ topological sector, where the lowest-energy solution we have found - for any value of the potential parameter $M$ turns out to be partially degenerate. That is, the red vortices (i.e., those of $\phi_{1}$ ) are degenerate, whereas the blue vortices (i.e., those of $\phi_{2}$ ) are nondegenerate. We did find a Skyrmion solution in this topological sector with nondegenerate vortices, which is a solution made of two $B=4$ cubes joined together. It turned out, however, to have a higher energy than the partially degenerate fullerene-like solution. It would be interesting if this is an exception, or there actually exists a lower-energy solution for the $B=8$ sector that we somehow did not find. As an argument in favor of this hypothesis, we found that the difference in energy between the nondegenerate and the partially degenerate Skyrmion solutions in the $B=4$ sector turned out to be extremely small. Therefore, increasing the potential parameter, $M$, may not be a viable technique in such a situation, as incredibly large values of $M$ could be needed for finding the lowest-energy state (if such a state exists).
Other approaches for searching for the lowest-energy state may be needed.

It would be interesting to study the case in which a "Josephson (or Rabi) term" $\phi_{1}^{*} \phi_{2}+$ c.c. is added to the potential. In the case of two-component BECs, this term induces a sine-Gordon soliton stretching between two types of vortices, exhibiting vortex confinement [37-44]. Several aspects of vortices in this case have been studied extensively, in particular, in two spatial dimensions, such as a vortex lattice [39], confinement [40,42], dynamics of vortices [41], collisions of vortices [44], as well as the phase structure [43]. In our case, two linked vortex lines will be connected by a minimal-surface soliton sheet, like a soap film.

In Ref. [2] we found that there exists a certain projection with which a baryon contains linked vortices. In this paper, we have shown that with a certain potential, those linked vortices can become physical.

\section{ACKNOWLEDGMENTS}

S. B. G. thanks the Outstanding Talent Program of Henan University for partial support. The work of S. B. G. is supported by the National Natural Science Foundation of China (Grant No. 11675223). The work of M.N. is supported in part by JSPS Grant-in-Aid for Scientific Research (KAKENHI Grant No. 18H01217).
[1] W. H. Thomson, On vortex motion, Trans. R. Soc. Edinburgh 25, 217 (1868).

[2] S. B. Gudnason and M. Nitta, Linking number of vortices as baryon number, Phys. Rev. D 101, 065011 (2020).

[3] T. Skyrme, A nonlinear field theory, Proc. R. Soc. A 260, 127 (1961).

[4] T. Skyrme, A unified field theory of mesons and baryons, Nucl. Phys. 31, 556 (1962).

[5] E. Witten, Global aspects of current algebra, Nucl. Phys. B223, 422 (1983).

[6] E. Witten, Current algebra, baryons, and quark confinement, Nucl. Phys. B223, 433 (1983).

[7] R. Battye, N. S. Manton, and P. Sutcliffe, Skyrmions and the alpha-particle model of nuclei, Proc. R. Soc. A 463, 261 (2007).

[8] R. A. Battye, N. S. Manton, P. M. Sutcliffe, and S. W. Wood, Light nuclei of even mass number in the skyrme model, Phys. Rev. C 80, 034323 (2009).

[9] S. Baldino, S. Bolognesi, S. B. Gudnason, and D. Koksal, Solitonic approach to holographic nuclear physics, Phys. Rev. D 96, 034008 (2017).

[10] J. Ruostekoski and J. Anglin, Creating Vortex Rings and Three-Dimensional Skyrmions in Bose-Einstein Condensates, Phys. Rev. Lett. 86, 3934 (2001).
[11] R. A. Battye, N. Cooper, and P. M. Sutcliffe, Stable Skyrmions in Two Component Bose-Einstein Condensates, Phys. Rev. Lett. 88, 080401 (2002).

[12] U. A. Khawaja and H. T. C. Stoof, Skyrmions in a ferromagnetic Bose-Einstein condensate, Nature (London) 411, 918 (2001).

[13] U. Khawaja and H. Stoof, Skyrmion physics in Bose-Einstein ferromagnets, Phys. Rev. A 64, 043612 (2001).

[14] C. Savage and J. Ruostekoski, Energetically Stable Particlelike Skyrmions in a Trapped Bose-Einstein Condensate, Phys. Rev. Lett. 91, 010403 (2003).

[15] J. Ruostekoski, Stable particlelike solitons with multiplyquantized vortex lines in Bose-Einstein condensates, Phys. Rev. A 70, 041601 (2004).

[16] S. Wuster, T. E. Argue, and C. M. Savage, Numerical study of the stability of Skyrmions in Bose-Einstein condensates, Phys. Rev. A 72, 043616 (2005).

[17] I. F. Herbut and M. Oshikawa, Stable Skyrmions in Spinor Condensates, Phys. Rev. Lett. 97, 080403 (2006).

[18] A. Tokuno, Y. Mitamura, M. Oshikawa, and I. F. Herbut, Skyrmion in spinor condensates and its stability in trap potentials, Phys. Rev. A 79, 053626 (2009). 
[19] T. Kawakami, T. Mizushima, M. Nitta, and K. Machida, Stable Skyrmions in SU(2) Gauged Bose-Einstein Condensates, Phys. Rev. Lett. 109, 015301 (2012).

[20] M. Nitta, K. Kasamatsu, M. Tsubota, and H. Takeuchi, Creating vortons and three-dimensional Skyrmions from domain wall annihilation with stretched vortices in BoseEinstein condensates, Phys. Rev. A 85, 053639 (2012).

[21] K. Kasamatsu, M. Tsubota, and M. Ueda, Vortices in multicomponent Bose-Einstein condensates, Int. J. Mod. Phys. B 19, 1835 (2005).

[22] S. B. Gudnason and M. Nitta, Effective field theories on solitons of generic shapes, Phys. Lett. B 747, 173 (2015).

[23] S. B. Gudnason and M. Nitta, Incarnations of Skyrmions, Phys. Rev. D 90, 085007 (2014).

[24] S. B. Gudnason and M. Nitta, Baryonic torii: Toroidal baryons in a generalized Skyrme model, Phys. Rev. D 91, 045027 (2015).

[25] E. Witten, Superconducting strings, Nucl. Phys. B249, 557 (1985).

[26] S. B. Gudnason and M. Nitta, Skyrmions confined as beads on a vortex ring, Phys. Rev. D 94, 025008 (2016).

[27] S. B. Gudnason and M. Nitta, Baryonic handles: Skyrmions as open vortex strings on a domain wall, Phys. Rev. D 98, 125002 (2018).

[28] M. Eto, K. Kasamatsu, M. Nitta, H. Takeuchi, and M. Tsubota, Interaction of half-quantized vortices in twocomponent Bose-Einstein condensates, Phys. Rev. A 83, 063603 (2011).

[29] K. Kasamatsu, M. Eto, and M. Nitta, Short-range intervortex interaction and interacting dynamics of halfquantized vortices in two-component Bose-Einstein condensates, Phys. Rev. A 93, 013615 (2016).

[30] A. Jackson, A. Jackson, A. Goldhaber, G. Brown, and L. Castillejo, A modified Skyrmion, Phys. Lett. B 154, 101 (1985).

[31] C. Adam, J. Sanchez-Guillen, and A. Wereszczynski, A Skyrme-type proposal for baryonic matter, Phys. Lett. B 691, 105 (2010).

[32] C. Adam, J. Sanchez-Guillen, and A. Wereszczynski, A BPS Skyrme model and baryons at large $N_{c}$, Phys. Rev. D 82, 085015 (2010).
[33] S. B. Gudnason and M. Nitta, A higher-order Skyrme model, J. High Energy Phys. 09 (2017) 028.

[34] S. B. Gudnason and J. M. Speight, Realistic classical binding energies in the $\omega$-Skyrme model, J. High Energy Phys. 07 (2020) 184.

[35] C. J. Houghton, N. S. Manton, and P. M. Sutcliffe, Rational maps, monopoles and Skyrmions, Nucl. Phys. B510, 507 (1998).

[36] C. Adam, C. Naya, J. Sanchez-Guillen, J. Speight, and A. Wereszczynski, Thermodynamics of the BPS Skyrme model, Phys. Rev. D 90, 045003 (2014).

[37] D. T. Son and M. A. Stephanov, Domain walls in twocomponent Bose-Einstein condensates, Phys. Rev. A 65, 063621 (2002).

[38] K. Kasamatsu, M. Tsubota, and M. Ueda, Vortex Molecules in Coherently Coupled Two-Component Bose-Einstein Condensates, Phys. Rev. Lett. 93, 250406 (2004).

[39] M. Cipriani and M. Nitta, Crossover Between Integer and Fractional Vortex Lattices in Coherently Coupled TwoComponent Bose-Einstein Condensates, Phys. Rev. Lett. 111, 170401 (2013).

[40] M. Tylutki, L. P. Pitaevskii, A. Recati, and S. Stringari, Confinement and precession of vortex pairs in coherently coupled Bose-Einstein condensates, Phys. Rev. A 93, 043623 (2016).

[41] L. Calderaro, A. L. Fetter, P. Massignan, and P. Wittek, Vortex dynamics in coherently coupled Bose-Einstein condensates, Phys. Rev. A 95, 023605 (2017).

[42] M. Eto and M. Nitta, Confinement of half-quantized vortices in coherently coupled Bose-Einstein condensates: Simulating quark confinement in a QCD-like theory, Phys. Rev. A 97, 023613 (2018).

[43] M. Kobayashi, M. Eto, and M. Nitta, Berezinskii-KosterlitzThouless Transition of Two-Component Bose Mixtures with Intercomponent Josephson Coupling, Phys. Rev. Lett. 123, 075303 (2019).

[44] M. Eto, K. Ikeno, and M. Nitta, Collision dynamics and reactions of fractional vortex molecules in coherently coupled Bose-Einstein condensates, arXiv:1912.09014 [Phys. Rev. Res. (to be published)]. 